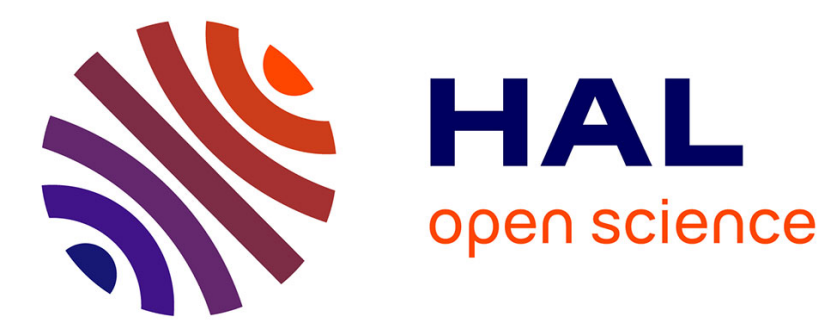

\title{
Packings of irregular polyhedral particles: Strength, structure, and effects of angularity
}

Emilien Azéma, Farhang Radjai, Frédéric Dubois

\section{To cite this version:}

Emilien Azéma, Farhang Radjai, Frédéric Dubois. Packings of irregular polyhedral particles: Strength, structure, and effects of angularity. Physical Review E : Statistical, Nonlinear, and Soft Matter Physics, 2013, 87 (062203), pp.1-15. 10.1103/PhysRevE.87.062203 . hal-00835121

\section{HAL Id: hal-00835121 \\ https://hal.science/hal-00835121}

Submitted on 18 Jun 2013

HAL is a multi-disciplinary open access archive for the deposit and dissemination of scientific research documents, whether they are published or not. The documents may come from teaching and research institutions in France or abroad, or from public or private research centers.
L'archive ouverte pluridisciplinaire HAL, est destinée au dépôt et à la diffusion de documents scientifiques de niveau recherche, publiés ou non, émanant des établissements d'enseignement et de recherche français ou étrangers, des laboratoires publics ou privés. 


\title{
Packings of irregular polyhedral particles: Strength, structure, and effects of angularity
}

\author{
Emilien Azéma, ${ }^{*}$ Farhang Radjai, ${ }^{\dagger}$ and Frédéric Dubois ${ }^{\ddagger}$ \\ Université Montpellier 2, CNRS, LMGC, Cc 048, Place Eugène Bataillon, F-34095 Montpellier cedex 05, France
}

(Received 12 January 2013; published 17 June 2013)

\begin{abstract}
We present a systematic numerical investigation of the shear strength and structure of granular packings composed of irregular polyhedral particles. The angularity of the particles is varied by increasing the number of faces from 8 (octahedronlike shape) to 596. We find that the shear strength increases with angularity up to a maximum value and saturates as the particles become more angular (below 46 faces). At the same time, the packing fraction increases to a peak value but declines for more angular particles. We analyze the connectivity and anisotropy of the microstructure by considering both the contacts and branch vectors joining particle centers. The increase of the shear strength with angularity is shown to be due to a net increase of the fabric and force anisotropies but at higher particle angularity a rapid falloff of the fabric anisotropy is compensated by an increase of force anisotropy, leading thus to the saturation of shear strength.
\end{abstract}

DOI: 10.1103/PhysRevE.87.062203

PACS number(s): 45.70.-n, 83.80.Fg, 61.43.-j

\section{INTRODUCTION}

The paradigm of sphere packings is commonly used to describe the structure of matter at different scales, from ordered atomic arrangements to disordered assemblies of colloidal particles. The sphere packing is also a model for granular materials when the interactions are governed by contact and friction. However, compared to real granular materials, the spherical shape of the particles is an enormous simplification. For example, it is classically well known in soil mechanics that the mechanical behavior of granular soils is strongly influenced by deviations of particles from spherical shape, described in terms of elongation, angularity, platiness, and other shape characteristics [1,2]. It is obvious that sphere packings are more easily amenable to numerical simulations and analysis. Moreover, it is often believed that, major ingredients such as frictional contact, dissipative collisions, steric exclusions, and structural disorder being equally present in packings of spherical and aspherical particles, the shape is a secondary factor that should not lead to significant amendment of our understanding of granular materials. The issue remains, nevertheless, as to the limits of deviation from spherical shape. For example, a packing of very elongated particles such as fibers is likely to behave very differently from a sphere packing. This holds true also for characteristics such as platiness and nonconvexity.

Recently, the packing fraction and shear strength of two-dimensional (2D) packings of different particle shapes were investigated in a collective work by discrete-element simulations [3]. It was found that, for different shapes, the shear strength is mainly controlled by a low-order common parameter describing particle shape by its deviation from circular shape. At the same time, the packing fraction increases with slightest deviation, reaches a peak value, and then declines for still larger deviation. This is an interesting observation as it means that an assembly of particles with large deviation from circular shape has a high shear strength despite a low packing fraction.

\footnotetext{
*emilien.azema@univ-montp2.fr

†ranck.radjai@univ-montp2.fr

${ }^{\ddagger}$ frederic.dubois@univ-montp2.fr
}

The nonmonotonic evolution of packing fraction in packings prepared by isotropic compression or sheared towards a steady shear state has been more extensively studied in the case of elongated particles [4-9]. Such particles are interesting since they give rise to a rich microstructure due to local and long-range orientational ordering of the particles [10-15], but also because they form a large class defined by a single parameter (the aspect ratio) having sphere as a special case (unit aspect ratio). This behavior was also observed in the case of nonconvex aggregates in 2D [16,17].

Angular shape represents basically a property of polygonal particles in 2D and polyhedral particles in 3D [18-29]. It is only very recently that systematic studies were reported on the maximal-density packings of monodisperse Platonic polyhedra [30-32] on one hand and for sheared packings composed of regular polygons on the other hand [33]. In this case, a nonlinear dependence of the shear strength and packing fraction has been evidenced by means of numerical simulations in 2D packings. The shear behavior and microstructure of granular materials composed of polyhedral particles was also investigated by comparing numerically a packing of polyhedral particles with a packing of similar particle size distribution and contact friction but with spherical particles [22,24]. The polyhedra packing is found to have a higher shear stress and dilatancy. The origin of these enhanced properties of the polyhedra packing was traced back to the properties of both packings in terms of branch vectors and contact forces. In particular, it was shown that the face-face contacts capture strong force chains, leading to a higher force anisotropy.

In this paper, we perform a systematic study of the effect of angularity by simulating different packings of irregular polyhedral particles with different numbers of faces but similar values of their other characteristics. The angularity increases as the number of faces declines from 596 (least angular shape) to 8 (most angular shape). We are interested in the packing properties in the dense state generated by isotropic compaction and the shear behavior and evolution of fabric parameters under axial compression as particle angularity is varied. The least angular particles are very close to spherical shape but, because of the large number of faces, the simulations take much more time. Simulating such particles makes it possible to evaluate directly the influence of small deviations from 
spherical shape. Ultimately, we would like to understand to what extent very angular particles are different from less angular particles.

In the following, the numerical procedures in the framework of the contact dynamics method are presented in Sec. II. In Sec. III, we focus on shear strength and packing fraction for different values of angularity. The microstructure is analyzed in Sec. IV in terms of connectivity, fabric anisotropy, and forces anisotropy. We conclude with a summary of the main findings of this work.

\section{NUMERICAL MODEL}

\section{A. Simulation of polyhedral particles}

The simulations were carried out by means of the contact dynamics (CD) method [27,34-36]. The CD method is based on implicit time integration of the equations of motion and a nonsmooth formulation of mutual exclusion and dry friction between particles. This method requires no elastic repulsive potential and no smoothing of the Coulomb friction law for the determination of forces. For this reason, the simulations can be performed with large time steps compared to molecular dynamics simulations. The unknown variables are particle velocities and contact forces. These are calculated at each time step by taking into account the conservation of momenta and the constraints due to mutual exclusion between particles and Coulomb friction. We use an iterative research algorithm based on a nonlinear Gauss-Seidel scheme. The uniqueness is not guaranteed for perfectly rigid particles in absolute terms. However, by initializing each step of calculation with the forces calculated in the preceding step, the set of admissible solutions shrinks to fluctuations which are basically below the numerical solution.

Angular polyhedral particles are constructed following a strict procedure in order to isolate and control precisely the shape. First, a set of $n_{v}$ vertices is randomly generated on a unit sphere. The convex hull of these points is created by associating three vertices for each face. This condition implies that the number $n_{f}$ of faces is simply given by $n_{f}=2 n_{v}-4$. Second, as illustrated in Fig. 1, the principal axis lengths $L_{p}$, $l_{p}$, and $h_{p}$ of the particle are calculated. The aspect ratios of a particle is then given by $L_{p} / h_{p}$ and $l_{p} / h_{p}$. In order to eliminate the effect of particle eccentricity, we impose that both ratios $L_{p} / h_{p}$ and $l_{p} / h_{p}$ be greater than 0.9 . This can be

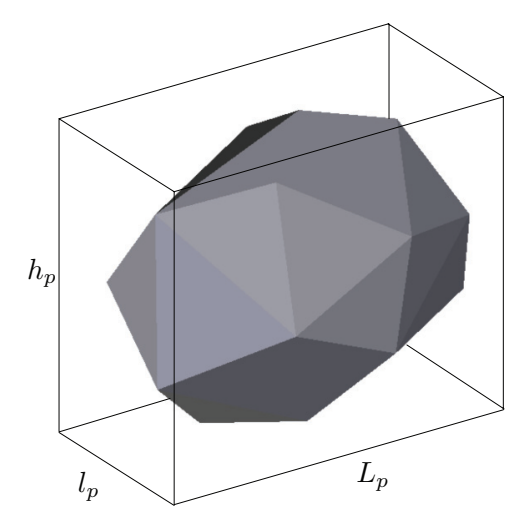

FIG. 1. (Color online) Definition of the aspect ratio.
TABLE I. Geometrical characteristics of numerical samples.

\begin{tabular}{lccccc}
\hline \hline Samples & $n_{f}$ & $\langle\alpha\rangle$ & $\left\langle L_{p} / h_{p}\right\rangle$ & $\left\langle l_{p} / h_{p}\right\rangle$ & $\Delta \alpha$ \\
\hline S1 & Spheres & 0 & 1 & 1 & 0 \\
S2 & 596 & 0.135 & 0.98 & 0.95 & $2.1 \times 10^{-3}$ \\
S3 & 176 & 0.248 & 0.97 & 0.94 & $2.1 \times 10^{-3}$ \\
S4 & 96 & 0.339 & 0.96 & 0.94 & $3.8 \times 10^{-3}$ \\
S5 & 46 & 0.496 & 0.93 & 0.95 & $7.7 \times 10^{-3}$ \\
S6 & 30 & 0.631 & 0.93 & 0.94 & $1.16 \times 10^{-2}$ \\
S7 & 20 & 0.772 & 0.93 & 0.91 & $1.66 \times 10^{-2}$ \\
S8 & 8 & 1.181 & 0.92 & 0.92 & $1.08 \times 10^{-2}$ \\
\hline \hline
\end{tabular}

evaluated also thanks to the sphericity parameter $\mathcal{S}$, defined as the ratio of the surface area of a sphere with the same volume as that of the given particle [37]. This means that, numerically, the sets of vertices are generated until this condition is satisfied. We define thus the angularity $\alpha$ of a polyhedral particle as the mean exterior angle between its touching faces. In this simple way, for a given aspect ratio, we can control the angularity of the particles with a single continuously variable shape parameter depending only on the number of faces $n_{f}$; see Table I.

Contact detection represents an important part of the discrete numerical modeling of polyhedra particles. The detection methods are split into different steps. First, a rough description of the particles leads to building a list of neighboring particle pairs. This first step is known as the "bounding box" method [38]. Then the distance between each pair of polyhedra is calculated by computing the separating plane through the so-called "common plane" method introduced by Cundall [39]. This is an iterative method based on the perturbation of the orientation of the normal vector. The process is initialized by a first guess based on the vector joining the centers of the two polyhedra. The main advantage of this method is to be quite fast when optimized as proposed by [18,40,41]. Note that, a dual approach based on separated axis, also known as the "shadow overlap" method, has been developed by Saussine et al. [23,24,28,33,38,42,43].

Finally, different situations may arise, such as grazing, separated, or penetrated contacts. For penetrated or ideal grazing contacts, different cases are possible, such as those shown in Fig. 2: contact point, contact line, or contact surface. Point contacts include face-vertex $(f v)$, edge-edge $(e e)$, vertex-vertex $(v v)$, or vertex-edge (ve) contacts. Note that $v v$ and $v e$ contacts are very rare. However, when they occur, the common plane method is able to give us the normal direction. Without any modification of the contact law, a contact line (or face-edge $f e$ ) can be represented by two points, whereas a flat contact (or face-face $f f$ ) can be replaced by three points since they involve an equivalent number of geometrical unilateral constraints. In the iterative procedure of determination of the contact forces and velocities, the points representing the contact between two particles are treated as independent points but the net resultant of the calculated forces is attributed to the contact with its application point located on the contact plane. In our simulations the average overlap between particles at different types of contact (simple, double, or triple) is $0.01 d$.

For our simulations, we used the LMGC90, which is a multipurpose software developed in Montpellier, capable 


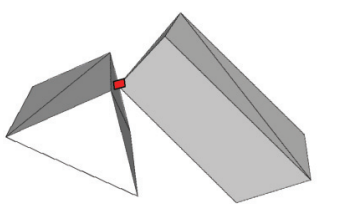

(a)

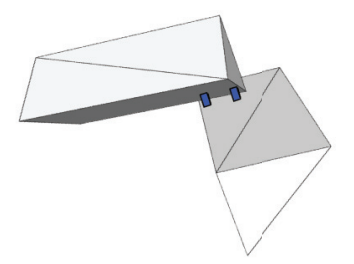

(b)
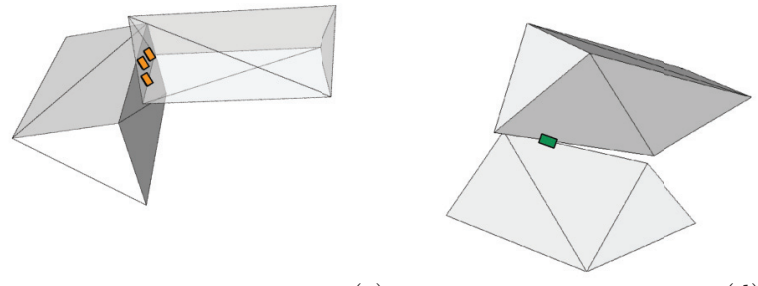

(c)

(d)

FIG. 2. (Color online) Different types of contacts between two polyhedra: (a) vertex to face, (b) edge to face, (c) face to face, (d) edge to edge.

of modeling a collection of deformable or nondeformable particles of various shapes by different algorithms [43-46].

\section{B. Sample generation}

We prepared seven different packings, each of them comprising 40000 irregular polyhedra with the same number $n_{f}$ of faces. The mean packing angularity $\langle\alpha\rangle$ is given by the mean angularity of the particles in the packing. We prepare one more packing composed of spheres. In order to avoid longrange ordering, we introduce also a weak size polydispersity by varying the diameter $d$ of the circumscribing sphere of the particles in the range of $\left[d_{\min }, 2 d_{\min }\right]$ with a uniform distribution of particle volume fractions. The geometrical characteristics of each sample are summarized in Table I. Note that the standard deviation of particle angularity $\Delta \alpha$ in each sample is used in all plots as the error bar on the angularity. Figure 3 displays several snapshots of the packings for different values of $\langle\alpha\rangle$ at the end of isotropic compaction (see below).

All samples are prepared according to the same protocol. A dense packing composed of spheres is first constructed by means of a layer-by-layer construction model based on simple geometrical rules [47]. The spherical particles are sequentially placed inside a box by searching a position of lowest gravitational potential energy so that each new particle is supported by three particles. For polyhedral particles, the same sphere packing is then used with each sphere serving as the circumscribing sphere of a polyhedral particle. The latter is inscribed with the given value of $\langle\alpha\rangle$ and a random orientation.

Following this geometrical process, each packing is compacted by isotropic compression inside a box of dimensions $L_{0} \times l_{0} \times H_{0}$ in which the left, bottom, and back walls are fixed and the top, right, and front walls are subjected to the same compressive stress $\sigma_{0}$ as illustrated in Fig. 4(a). The gravity $g$ and friction coefficient between particles and with (a)

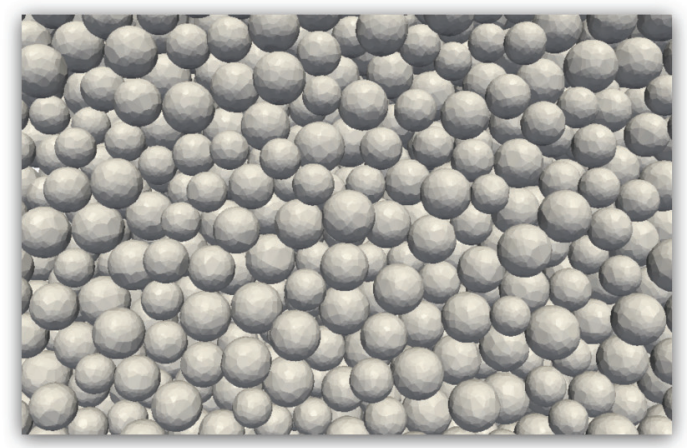

(b)

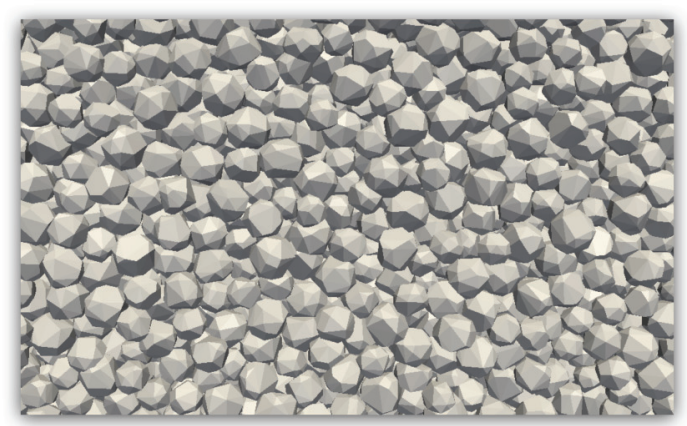

(c)

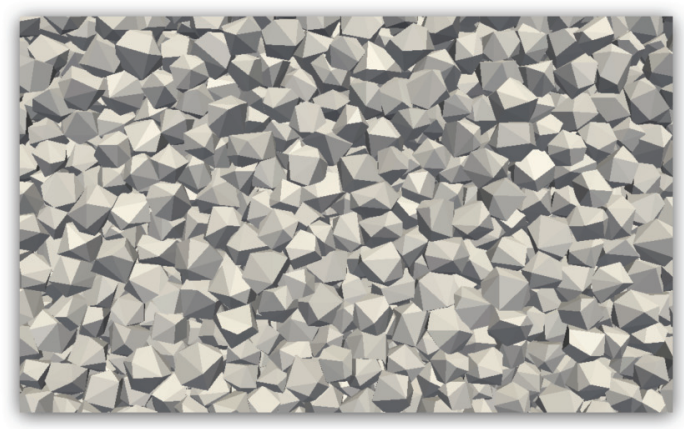

(d)

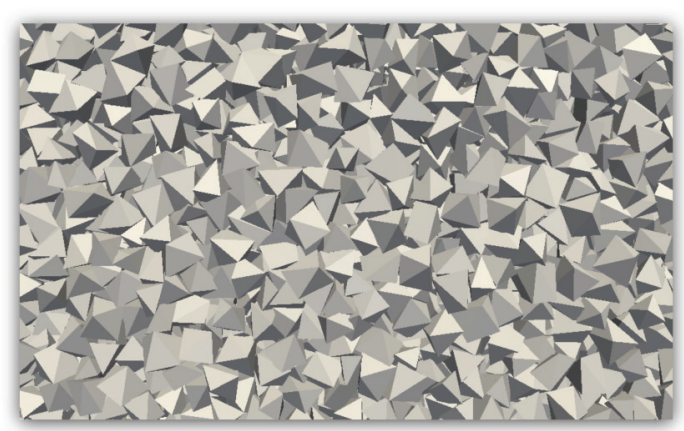

FIG. 3. (Color online) Snapshot of a portion of the packings at the end of isotropic compression with $n_{f}=596$ (a), $n_{f}=46$ (b), $n_{f}=20$ (c), and $n_{f}=8$ (d).

the walls are set to zero during isotropic compression in order to obtain isotropic dense packings.

The isotropic samples obtained in this way are subjected to vertical compression by downward displacement of the top wall at a constant velocity $v_{z}$ for a constant confining stress $\sigma_{0}$ exerted on the lateral walls; see Fig. 4(b). The friction coefficient between particles is set to $\mu=0.4$ and to zero with the walls. Since we are interested in quasistatic behavior, the shear rate should be such that the kinetic energy supplied by 
(a)

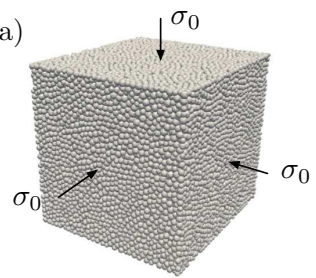

(b)

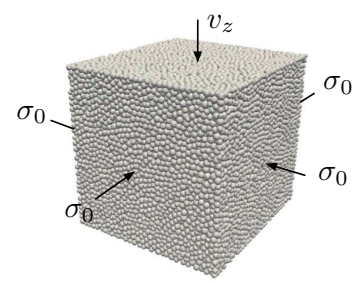

FIG. 4. (Color online) Boundary conditions for (a) isotropic and (b) triaxial compression.

shearing is negligible compared to the static pressure. This can be formulated in terms of an inertia parameter $I$ defined by [48]

$$
I=\dot{\varepsilon} \sqrt{\frac{m}{d \sigma_{0}}},
$$

where $\dot{\varepsilon}=\dot{z} / z$ and $m$ is the particle mass. The quasistatic limit is characterized by the condition $I \ll 1$. In our simulations, $I$ remains below $10^{-3}$ during shear. Note that video samples of the simulations analyzed in this paper can be found following this link: www.cgp-gateway.org/ref016.

\section{MACROSCOPIC BEHAVIOR}

\section{A. Macroscopic parameters}

We need to evaluate the stress tensor and packing fraction during deformation from the simulation data. For the stress tensor, we start with the internal moment $\boldsymbol{M}^{i}$ of each particle $i$ defined by [49]

$$
M_{\gamma \delta}^{i}=\sum_{c \in i} f_{\gamma}^{c} r_{\delta}^{c},
$$

where $f_{\gamma}^{c}$ is the (only) $\gamma$ component of the force exerted on particle $i$ at the contact $c, r_{\delta}^{c}$ is the $\delta$ component of the position vector of the same contact $c$, and the summation runs over all contact neighbors of particle $i$ (noted briefly by $c \in i$ ). The average stress tensor $\sigma$ in the volume $V$ of the granular assembly is given by the sum of the internal moments of individual particles divided by the total volume [49],

$$
\boldsymbol{\sigma}=\frac{1}{V} \sum_{i \in V} \boldsymbol{M}^{i}=\frac{1}{V} \sum_{c \in V} f_{\gamma}^{c} \ell_{\delta}^{c},
$$

where $\ell^{c}$ is the branch vector joining the centers of the two touching particles at the contact point $c$. We note that the first summation runs over all particles, whereas the second summation involves the contacts, each contact appearing only once.

Under triaxial conditions with vertical compression, we have $\sigma_{1} \geqslant \sigma_{2}=\sigma_{3}$, where the $\sigma_{\alpha}$ are the stress principal values. The mean stress $p$ and stress deviator $q$ are defined by

$$
\begin{gathered}
p=\left(\sigma_{1}+\sigma_{2}+\sigma_{3}\right) / 3, \\
q=\left(\sigma_{1}-\sigma_{3}\right) / 3 .
\end{gathered}
$$

For our system of perfectly rigid particles, the stress state is characterized by the mean stress $p$ and the normalized shear stress $q / p$.

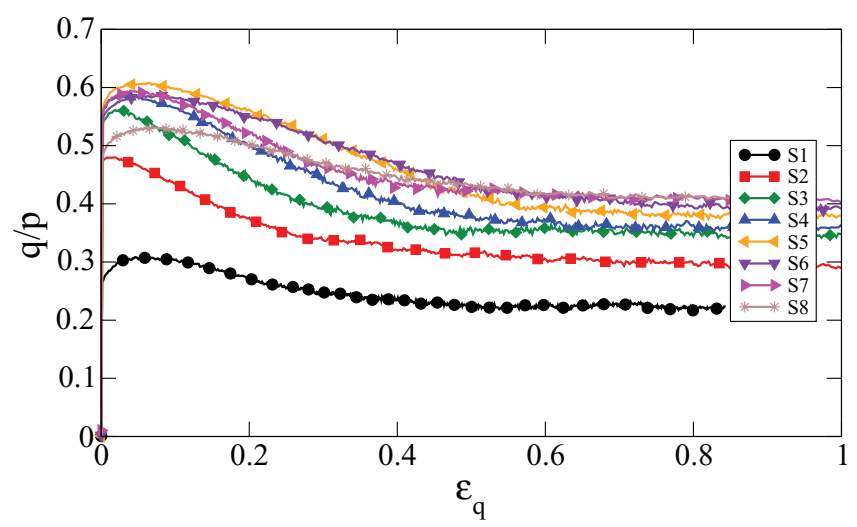

FIG. 5. (Color online) Normalized shear stress $q / p$ as a function of cumulative shear strain $\varepsilon_{q}$ for each sample.

The cumulative strain components $\varepsilon_{\alpha}$ are defined by

$$
\begin{gathered}
\varepsilon_{1}=\int_{H_{0}}^{H} \frac{d H^{\prime}}{H^{\prime}}=\ln \left(1+\frac{\Delta H}{H_{0}}\right), \\
\varepsilon_{2}=\int_{L_{0}}^{L} \frac{d L^{\prime}}{L^{\prime}}=\ln \left(1+\frac{\Delta L}{L_{0}}\right), \\
\varepsilon_{3}=\int_{l_{0}}^{l} \frac{d l^{\prime}}{l^{\prime}}=\ln \left(1+\frac{\Delta l}{l_{0}}\right),
\end{gathered}
$$

where $H_{0}, l_{0}$, and $L_{0}$ are the initial height, width, and length of the simulation box, respectively, and $\Delta H=H_{0}-H, \Delta l=$ $l_{0}-l$, and $\Delta L=L_{0}-L$ are the corresponding cumulative displacements. The volumetric strain is given by

$$
\varepsilon_{p}=\varepsilon_{1}+\varepsilon_{2}+\varepsilon_{3}=\int_{V_{0}}^{V} \frac{d V^{\prime}}{V^{\prime}}=\ln \left(1+\frac{\Delta V}{V_{0}}\right),
$$

where $V_{0}$ is the initial volume and $\Delta V=V-V_{0}$ is the total volume change. The cumulative shear strain is defined by

$$
\varepsilon_{q} \equiv \varepsilon_{1}-\varepsilon_{3} .
$$

We note that the choice of the deviatoric stress variable $q$ in Eq. (5) with a prefactor $1 / 3$ results from the requirement that the total power $\dot{W}=\sigma_{1} \dot{\varepsilon}_{1}+\sigma_{2} \dot{\varepsilon}_{2}+\sigma_{3} \dot{\varepsilon}_{3}$ should be expressed as a sum of the products of the volumetric and deviatoric conjugate variables $\dot{W}=p \dot{\varepsilon}_{p}+2 q \dot{\varepsilon}_{q}$.

\section{B. Shear strength and dilatancy}

Figure 5 shows the normalized shear stress $q / p$ as a function of shear strain $\varepsilon_{q}$ for all values of $\langle\alpha\rangle$. The jump observed at $\varepsilon_{q}=0$ reflects both the rigidity of the particles and high initial packing fraction of the samples (see below). In all cases, the shear stress passes by a peak before relaxing to a stress plateau corresponding to the so-called "residual state" in soil mechanics $[1,50]$. We observe also that the value of shear strength at the peak and residual state, respectively $(q / p)^{+}$and $(q / p)^{*}$, varies with particle's angularity. Figure 6(a) shows the variation of $(q / p)^{+}$and $(q / p)^{*}$ as a function of $\langle\alpha\rangle$. We see that $(q / p)^{+}$increases with $\langle\alpha\rangle$ up to a maximum value and then decreases as particles become more angular. In contrast, in the residual state $(q / p)^{*}$ increases with $\langle\alpha\rangle$ from 0.2 for spheres but saturates to $\simeq 0.4$ for the most angular shape. 

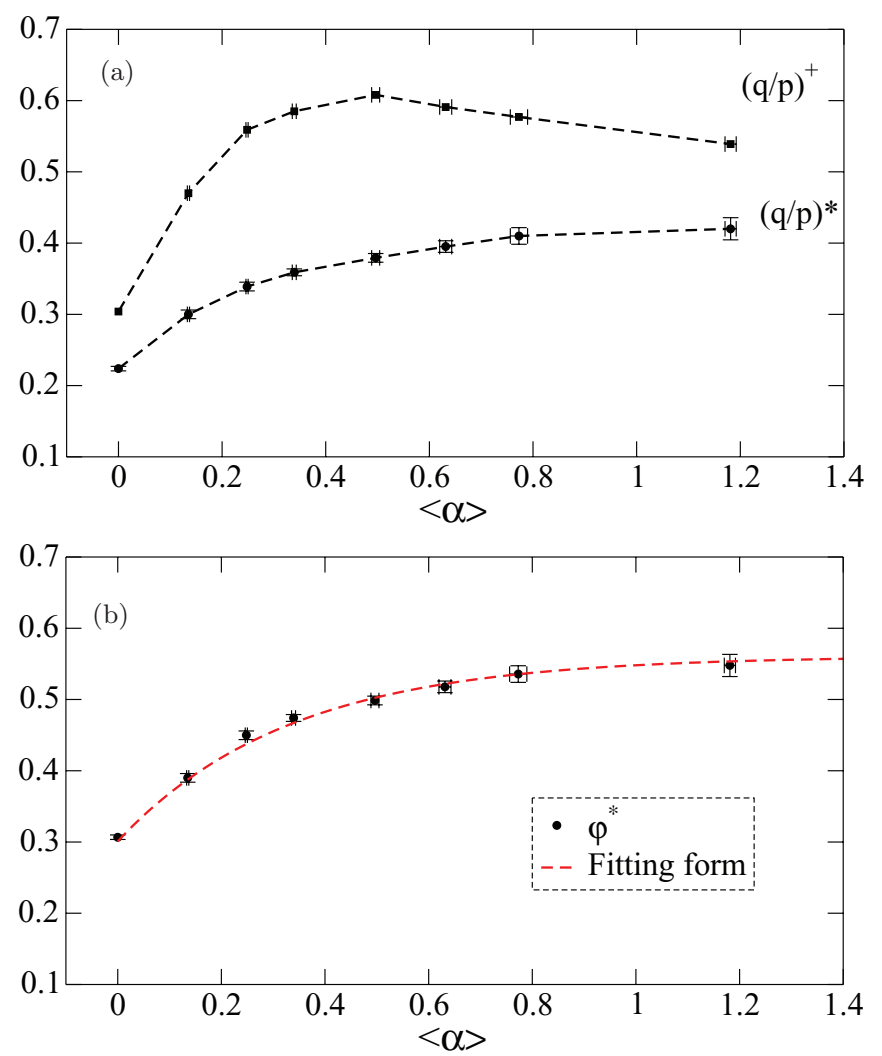

FIG. 6. (Color online) Normalized shear stress at peak and averaged in the residual state (a) and internal angle of friction $\varphi^{*}$ together with an exponential fit (b) as a function of $\langle\alpha\rangle$. Error bars represent the standard deviation in the residual state.

For triaxial geometry, the internal angle of friction $\varphi^{*}$, representing the shear strength of the material, is defined from the mean value $(q / p)^{*}$ of the normalized shear stress in the residual state by [1]

$$
\sin \varphi^{*}=\frac{3(q / p)^{*}}{2+(q / p)^{*}} .
$$

Figure 6(b) shows the variation of $\varphi^{*}$ as a function of $\langle\alpha\rangle$. As for $(q / p)^{*}$, we see that the friction angle increases from $\varphi_{0}^{*} \simeq 0.3$ and tends asymptotically to $\varphi_{1}^{*} \simeq 0.5$. The data are well fit to an exponential function,

$$
\varphi^{*}=\varphi_{0}^{*}+\left(\varphi_{1}^{*}-\varphi_{0}^{*}\right)\left(1-e^{-\alpha / \alpha_{c}}\right),
$$

with $\alpha_{c} \simeq 0.4$.

The fast increase of $\varphi^{*}$ with $\langle\alpha\rangle$ and its saturation is rather unexpected as it indicates that small deviations from spherical shape have much stronger effect on $\varphi^{*}$ than the larger variations of angularity (in the range of low numbers of faces). Heuristically, this effect may be understood by remembering that the particles may interact at two contacts (face-edge) or three contacts (face-face), so that the kinematic constraint due to such contacts increases even if the contact surface is small, as, for example, the case of polyhedra having 596 faces. This point is discussed in more detail when we analyze the microstructure.

Figure 7 displays the cumulative volumetric strain $\varepsilon_{p}$ as a function of $\varepsilon_{q}$ for all values of $\langle\alpha\rangle$. Starting with an initially dense state, all packings dilate and hence the volume increases.

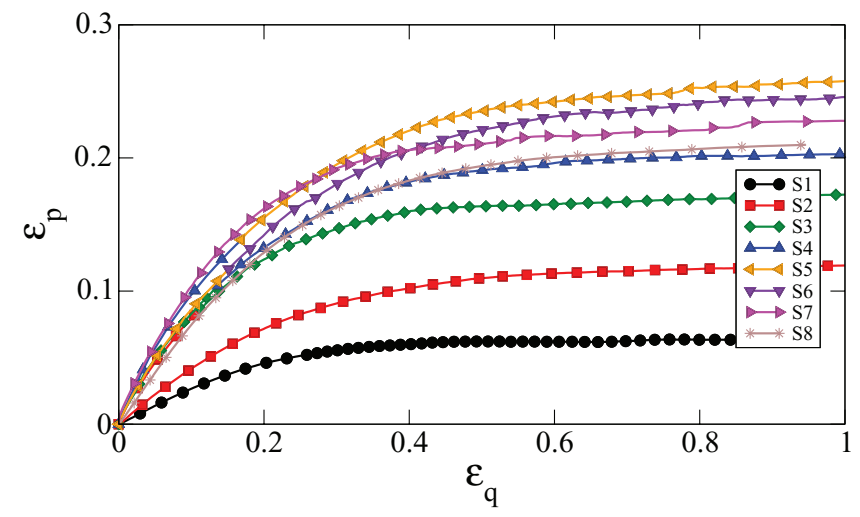

FIG. 7. (Color online) Cumulative volumetric strain $\varepsilon_{p}$ as a function of shear strain $\varepsilon_{q}$ for each samples.

A careful analysis of the velocity field indicates that the volume change is inhomogeneous and involves nonpersistent shear bands passing through different parts of the samples. For this reason, the shear strain $\varepsilon_{q}$ required to reach the critical state depends on the evolution of shear bands as well as the angularity. According to our data, it may be considered that the critical state with isochoric deformation is reached with a good approximation for all packings at $\varepsilon_{q} \simeq 0.6$.

Figure 8 displays the packing fraction $v$ as a function of $\langle\alpha\rangle$ at the initial state $\left(v^{0}\right)$ and averaged in the critical state $\left(v^{*}\right)$. It is remarkable that initially the packing fraction increases with $\langle\alpha\rangle$ up to a maximum value for $\langle\alpha\rangle \simeq 0.5$ and then declines as $\langle\alpha\rangle$ increases. We note that packing fractions as large as $v_{0} \simeq 0.75$ can be obtained in the initial state. A similar nonmonotonic behavior was observed for packings of ellipses, ellipsoidal, and platy particles with varying aspect ratios [4-7,51]. Recently, using molecular dynamic simulations, the maximally random jammed (MRJ) packings were also analyzed with "superballs" [52] whose shapes, defined by a single parameter $k \in[0, \infty]$, vary continuously from octahedronlike shapes $(k<1)$ to cubelike shapes $(k>1)$ and with Platonic polyhedra [31,32]. In both cases, the variation of packing fraction is also found to be nonmonotonic with $k$ or with the number of faces of the polyhedra. In particular, the packing fraction for octahedral particle shape (i.e., $k=0.85$ for superballs) or for regular

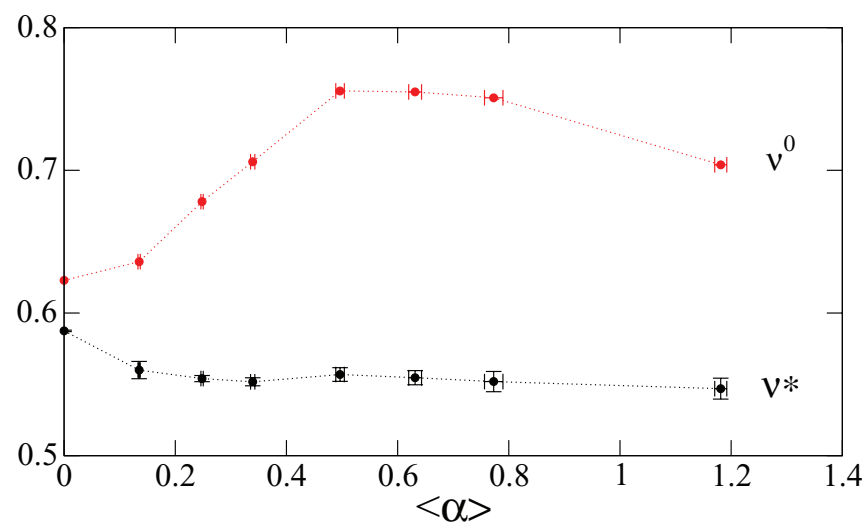

FIG. 8. (Color online) Packing fraction $v_{0}$ at the initial state (before shearing) and $v^{*}$ averaged in the residual state as a function of $\langle\alpha\rangle$. Error bars represent the standard deviation in the residual state. 
(a)

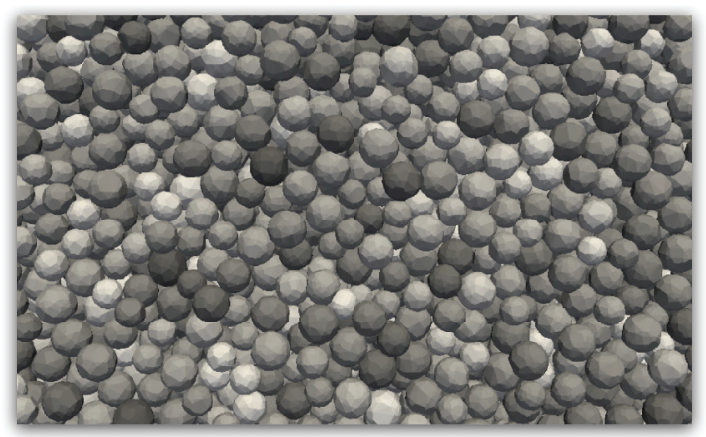

(b)

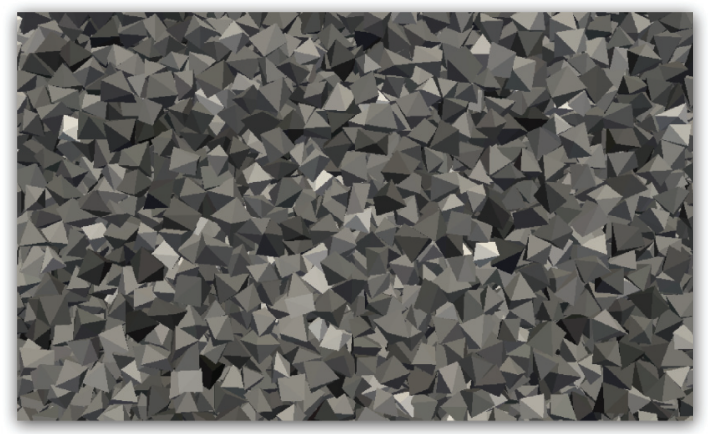

FIG. 9. Gray level map of particle connectivities for S3 (a) and S8 (b) in the residual state. Color intensity is proportional to coordination number.

polyhedral shapes is nearly equal to 0.68 , which is similar to what we obtain with our irregular octahedral particles (sample S8) by means of CD simulations.

A series of discrete element simulations reported recently with different particles shapes indicate that the nonmonotonic evolution of the packing fraction as a function of a shape parameter is a generic behavior [3]. The initial rapid increase of the packing fraction reveals that excluded-volume effects are not the prevailing mechanism at low angularity $\langle\alpha\rangle$. In this limit, slight deviations from spherical shape have strong space-filling effect on the packing although the excluded volume increases at the same time and becomes dominant for the higher angularities. In contrast, the packing fraction $v^{*}$ decreases from 0.58 (sphere) to 0.54 (octahedron).

The fact the packing fraction follows a trend opposite to that of the shear strength with increasing angularity is a somewhat counterintuitive finding, since it is often assumed that the shear strength in granular materials increases with the packing fraction. Our results for angular particles extend recent findings based on 2D simulations of elongated [8,9], polygonal [33], and nonconvex [16,17] particle shapes.

\section{GRANULAR MICROSTRUCTURE}

\section{A. Topology of the contact network}

A typical gray-level map of particle connectivity is shown in Fig. 9 for two values of $\alpha$ in the residual state. The packings of more angular particles are more connected. The coordination number $z$ in the initial state and its average value in the residual state are plotted as a function of $\langle\alpha\rangle$ in Fig. 10(a). The initial value $z_{0} \simeq 6$ corresponds to a frictionless packing of spherical particles in the isostatic state. Indeed, sphere rotations being
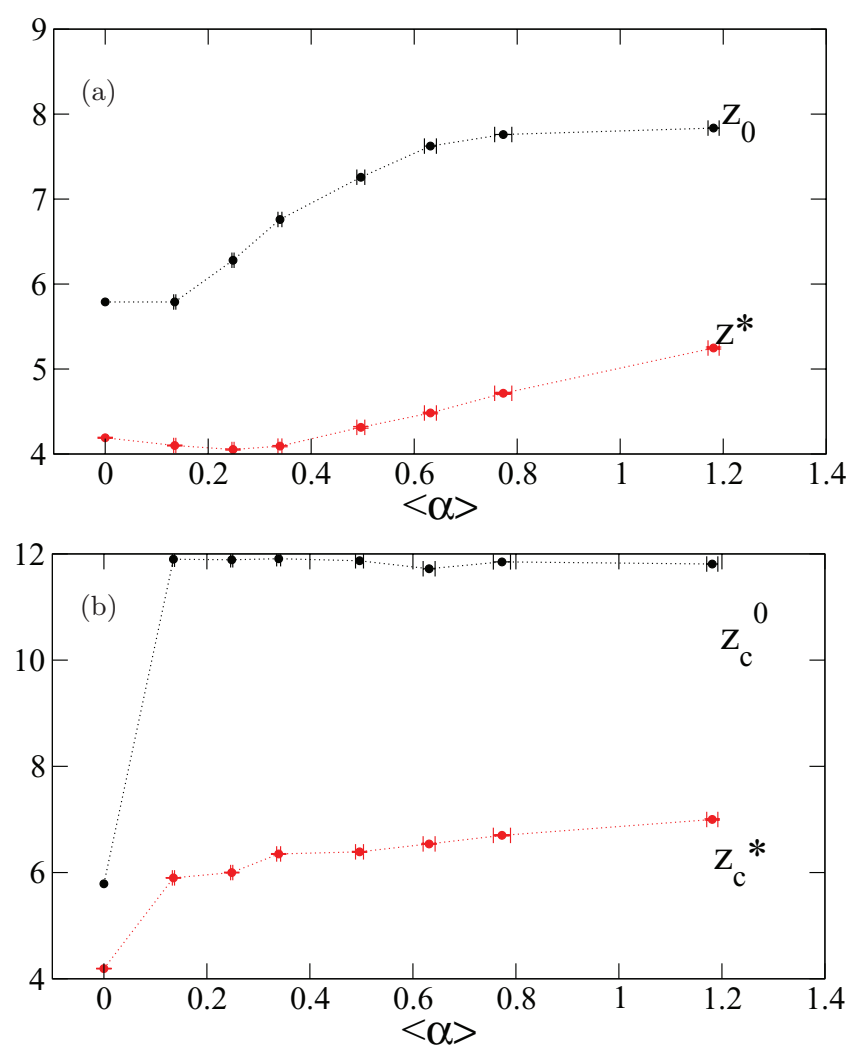

FIG. 10. (Color online) Initial and residual values of the coordination number as a function of $\langle\alpha\rangle$. Error bars represent the standard deviation in the residual state.

immaterial, the isostatic condition $z=2 d_{f}$, where $d_{f}$ is the number of degrees of freedom, implies three independent constraints (normal forces), which amounts to a coordination number 6 . As $\langle\alpha\rangle$ increases, $z_{0}$ increases and tends to a plateau value $\sim 8$.

These polyhedral shapes seem to violate the isostatic condition because for frictionless aspherical particles the rotations should be considered as effective degrees of freedom and a similar counting argument leads to a coordination number of 12. It has been found that ellipses, ellipsoids [53], and tetrahedra [54] have an average jamming contact number below their respective isostatic values (so "underconstrained"). The departure of ellipsoids from isostatic behavior has been attributed to the presence of floppy modes, which provide vanishing restoring force, whereas for tetrahedra it has been attributed to the varying degrees of rotational constraint by discrete contact topologies (e.g., vertex-face, edge-edge, edge-face, and face-face contacts).

However, remembering that face-edge and face-face contacts between polyhedra are equivalent to two and three contact points (involving thus, respectively, two and three unilateral constraints), we distinguish the coordination number $z$ (number of contact neighbors), from the connectivity numberz defined as the mean number of contacts per particle. We have

$$
\frac{z_{c}}{z}=k_{e e}+k_{f v}+2 k_{f e}+3 k_{f f},
$$

where $k_{f f}, k_{f e}, k_{f v}$, and $k_{e} e$ are the proportions of $f f, f e$, $f v$, and $e e$ contacts. Note that for spheres we have $z=z_{c}$. In 

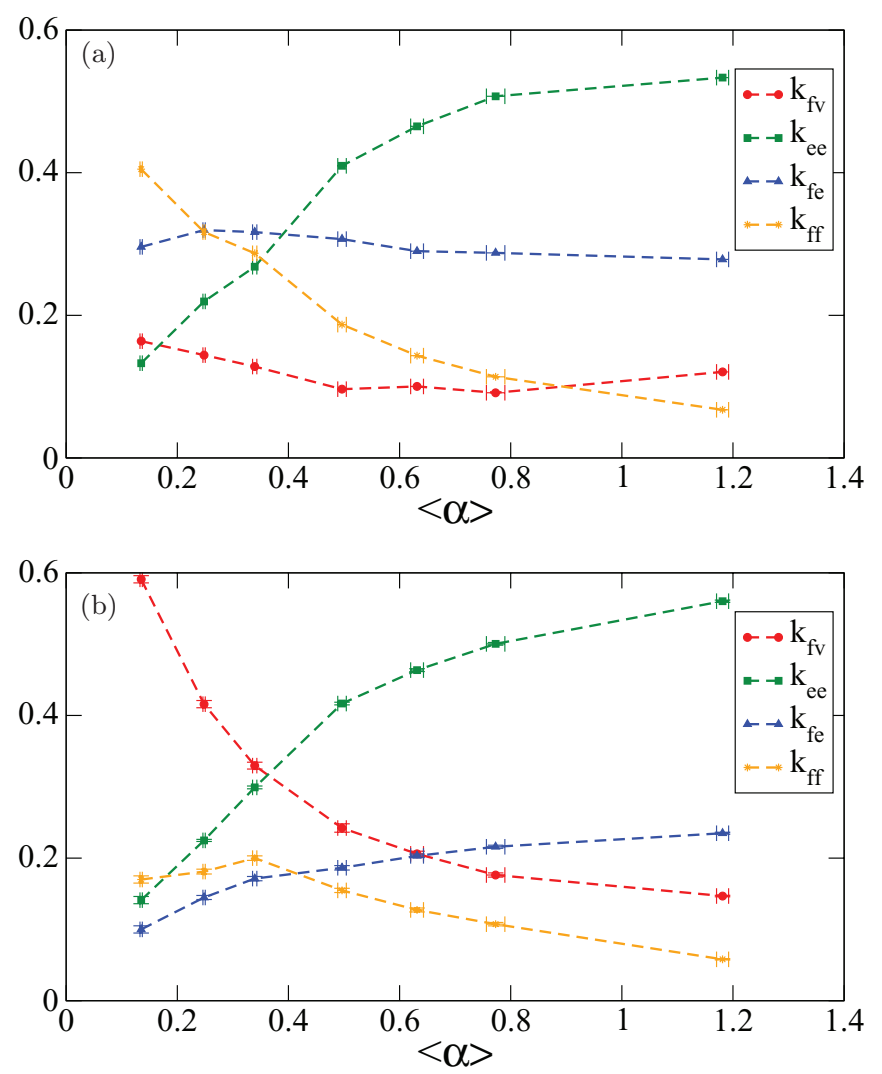

FIG. 11. (Color online) Proportions of $f v, e e, f e$, and $f f$ contacts as a function of $\langle\alpha\rangle$ in initial (a) and residual (b) states. The error bars represent the standard deviation in the residual state.

Fig. 10(b) we have plotted $z_{c}$ in both isotropic and residual states for all values of $\langle\alpha\rangle$. We see that $z_{c}^{0}$ jumps from 6 for spheres to $\sim 12$ for $\langle\alpha\rangle>0$, which is in full agreement with the isostatic nature of our initial packings. This observation indicates that aspherical particles are not underconstrained, and in numerical simulations the complex contacts can be represented by a set of point contacts. A similar result was also obtained by Saint-Cyr et al. in 2D with nonconvex particles [17].

In the residual state $z$ and $z_{c}^{*}$ are lower but both increase from 4 (spheres) to 5.5 and 7, respectively, as angularity increases. The effect of increasing angularity is therefore expressed by an increasing number of complex contacts as well as an increasing number of neighbors per particle, and thus for large $\langle\alpha\rangle$ the packings are loose but well connected. A similar behavior was found with elongated, nonconvex, and polygonal particles in $2 \mathrm{D}[8,17,55]$. Hence, the increase of shear strength with angularity seems to be linked more fundamentally with the increase of network connectivity rather than packing fraction, which varies rather nonmonotically with angularity.

To get further insight into the properties of the contact network, we plot in Fig. 11(a) the proportions of different contact types in the initial isostatic and residual states as a function of $\langle\alpha\rangle$. Interestingly, we observe that the proportion of $f v$ and $f e$ contacts in the isostatic state are nearly independent of $\langle\alpha\rangle$, and we have $k_{f e} \sim 2 k_{f v} \sim 0.3$. In contrast, $k_{f f}$ decreases from 0.4 to 0.1 and at the same time $k_{e e}$ increases from $\sim 0$ to 0.55 as a function of angularity. Hence, as particle angularity

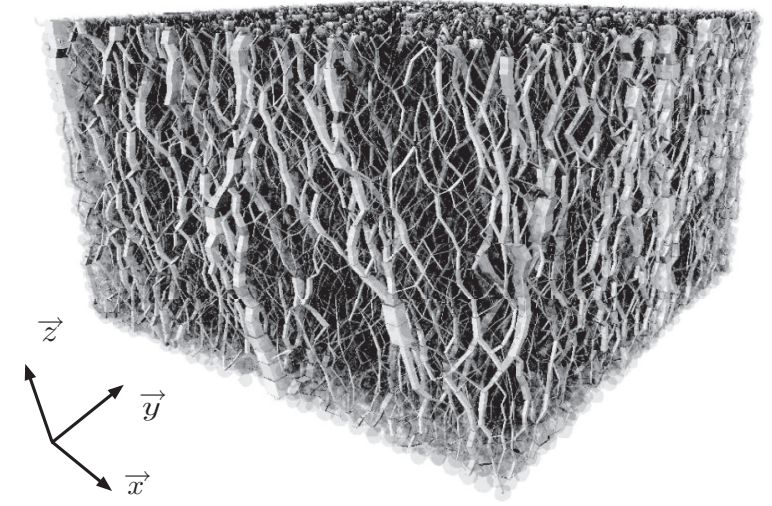

FIG. 12. Snapshot of radial forces in packing for $S 4$ in the steady state. Line thickness is proportional to the radial force.

increases, the packing changes from a network dominated by $f f$ contacts to a network dominated by ee contacts. Furthermore, remarking that $k_{e e}+k_{f v}+k_{f f}+k_{f e}=1$ in Eq. (13), we have the following relation between $z$ and $z_{c}$ :

$$
z_{c}=\left(1+k_{f e}+2 k_{f f}\right) z .
$$

This shows that in the initial state where $z_{c} \simeq 12$ the loss of face-face contacts is compensated by the increase of $z$. In the residual state, the proportion of $f v$ contacts is higher than in the isostatic state but it decreases with angularity from 0.6 to 0.2 . In contrast, we observe a loss of $f f$ and $f e$ contacts in the residual state compared to the initial isotropic state for all values of $\langle\alpha\rangle$.

\section{B. Anisotropies and origins of strength}

The above description of the microstructure in terms of the average coordination and connectivity numbers between polyhedra provides a basic picture of the effect of shape angularity. However, this picture is clearly insufficient since the contact and force networks are generically anisotropic as shown in Fig. 12, where a typical map of "radial" forces, defined as the components of contact forces along the branch vectors, is displayed in the sample $S 4$. In this section, we would like to evaluate these anisotropies, their relation with shear strength, and their evolution with particle angularity.

\section{Integral expression of the stress tensor}

The stress tensor defined by Eq. (3) is an arithmetic mean involving the branch vectors $\ell$ and contact force vectors $f$. Hence, it can be expressed as an integral through the joint probability density $P_{\ell f}$ of the force and branch vectors,

$$
\sigma_{\gamma \delta}=n_{c} \int f_{\gamma} \ell_{\delta} P_{\ell f} d \boldsymbol{f} d \boldsymbol{\ell},
$$

where $n_{c}$ is the number density of contacts.

The components of the branch vector and contact force for each pair of particles can be projected onto the local contact frame $(\boldsymbol{n}, \boldsymbol{t}, \boldsymbol{s})$, where $\boldsymbol{t}$ is an orthonormal unit vector oriented along the tangential force, and $s$ is defined such that the triad $(\boldsymbol{n}, \boldsymbol{t}, \boldsymbol{s})$ is an orthonormal basis; see Fig. 13(a). We have

$$
\boldsymbol{f}=f_{n} \boldsymbol{n}+f_{t} \boldsymbol{t}, \quad \boldsymbol{\ell}=\ell_{n} \boldsymbol{n}+\ell_{t} \boldsymbol{t}+\ell_{s} \boldsymbol{s},
$$



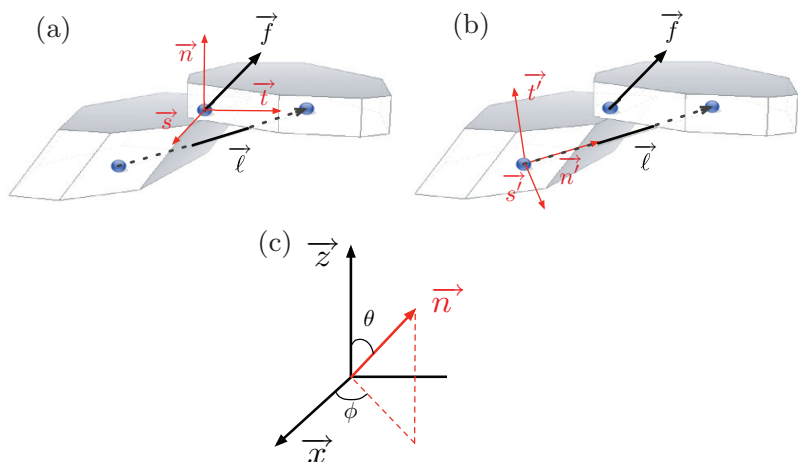

FIG. 13. (Color online) (a) Contact frame $(\boldsymbol{n}, \boldsymbol{t}, \boldsymbol{s})$, (b) branch vector frame $\left(\boldsymbol{n}^{\prime}, \boldsymbol{t}^{\prime}, \boldsymbol{s}^{\prime}\right),(\mathrm{c})$ azimutal $(\phi)$ and radial $(\theta)$ angles.

where $f_{n}$ and $f_{t}$ are the normal and tangential components of the contact force, and $\ell_{n}, \ell_{t}$ and $\ell_{s}$ are the components of the branch vector. We note that only for disks or spherical particles we have $\boldsymbol{\ell}=\boldsymbol{\ell} \boldsymbol{n}$, where $\ell$ is the length of the branch vector.

A similar decomposition can be applied also to the "neighborhood network" defined by the branch vector frames $\left(\boldsymbol{n}^{\prime}, \boldsymbol{t}^{\prime}, \boldsymbol{s}^{\prime}\right)$, where $\boldsymbol{n}^{\prime}$ is the unit vector along the branch vector; see Fig. 13(b). We express the components of the branch vector and contact force also in this frame by orienting the pair $\left(\boldsymbol{t}^{\prime}, \boldsymbol{s}^{\prime}\right)$ such that the component of the force along $\boldsymbol{s}^{\prime}$ vanishes. We have

$$
\ell=\ell_{n^{\prime}} \boldsymbol{n}^{\prime}, \quad \boldsymbol{f}=f_{n^{\prime}} \boldsymbol{n}^{\prime}+f_{t^{\prime}} \boldsymbol{t}^{\prime},
$$

where $f_{n^{\prime}}$ and $f_{t^{\prime}}$ are the radial and orthoradial components of the contact force, and $\ell_{n^{\prime}}=\ell$.

The orientations of the orthonormal local basis attached to the contact or to the branch vector can be parametrized by the angles $\theta$ and $\phi$ :

$$
\begin{aligned}
\boldsymbol{n} & =(\cos \theta, \sin \theta \cos \phi, \sin \theta \sin \phi), \\
\boldsymbol{t} & =(-\sin \theta, \cos \theta \cos \phi, \cos \theta \sin \phi), \\
\boldsymbol{s} & =(0,-\sin \phi, \cos \phi),
\end{aligned}
$$

where $\Omega=(\theta, \phi)$ is the solid angle that defines the orientation of $\boldsymbol{n}$ or $\boldsymbol{n}^{\prime}$ as illustrated in Fig. 13(c). Introducing the force and branch vector components in Eq. (15) and integrating with respect to $\phi$, we get the following expressions for the two independent invariants of the stress tensor in the contact frame:

$$
\begin{aligned}
p= & \frac{n_{c}}{3}\left\langle f_{n} \ell_{n}+f_{t} \ell_{t}\right\rangle, \\
q= & \frac{n_{c}}{6}\left\langle f_{n} \ell_{n}\left(3 \cos ^{3} \theta-1\right)-3\left(f_{t} \ell_{t} \cos ^{2} \theta\right)\right. \\
& \left.-\frac{3}{2}\left(f_{n} \ell_{t}+f_{t} \ell_{n}\right) \sin 2 \theta\right\rangle .
\end{aligned}
$$

In this expression, the average $\langle\cdots\rangle$ involves only the angle $\theta$ through the measure $P(\theta) \sin \theta d \theta d f_{n} d f_{t}, d \ell_{n}, d \ell_{t}$. Note that, due to the axial symmetry of the system, the $s$ components do not appear. In the same way, we get the following expressions in the branch-vector frame:

$$
\begin{aligned}
& p=\frac{n_{c}}{3}\left\langle f_{n^{\prime}} \ell\right\rangle, \\
& q=\frac{n_{c}}{6}\left\langle f_{n^{\prime}} \ell\left(3 \cos ^{3} \theta-1\right)-\frac{3}{2}\left(f_{t^{\prime}} \ell\right) \sin 2 \theta\right\rangle .
\end{aligned}
$$

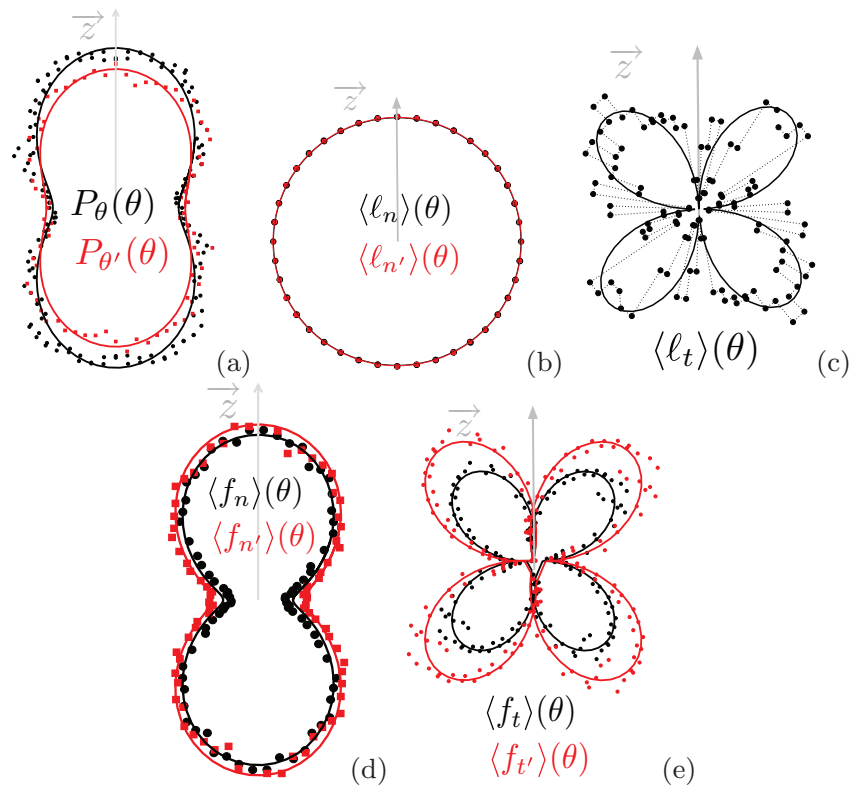

FIG. 14. (Color online) Polar representation of the functions $P_{\bar{\theta}}(\theta)(\mathrm{a}),\left\langle\ell_{\bar{n}}\right\rangle(\theta)$ (b), $\left\langle\ell_{t}\right\rangle(\theta)(\mathrm{c}),\left\langle f_{\bar{n}}\right\rangle(\theta)(\mathrm{d})$, and $\left\langle f_{\bar{t}}\right\rangle(\theta)$ (e) (symbols) and with the corresponding harmonic approximations (solid lines) for 56 .

\section{3D Harmonic representation of the microstructure}

In order to extract useful information from the expressions given in Eqs. (19) and (20), we can integrate out all force and branch components and keep only the dependence of the force and branch-vector components with respect to $\theta$. Let $\mathcal{S}(\Omega)$ be the set of contact vectors or branch vectors pointing in the direction $\Omega$ up to a solid angle $d \Omega$. From our numerical data we can evaluate the probability density functions $P_{\Omega}(\Omega)$ and $P_{\Omega^{\prime}}(\Omega)$ of contact orientations and branch vector orientations, respectively, as well as the angular averages of the force components $\left\langle f_{n}\right\rangle(\Omega),\left\langle f_{t}\right\rangle(\Omega),\left\langle f_{n^{\prime}}\right\rangle(\Omega)$, and $\left\langle f_{t^{\prime}}\right\rangle(\Omega)$ and branch vector components $\left\langle\ell_{n}\right\rangle(\Omega),\left\langle\ell_{t}\right\rangle(\Omega)$, and $\left\langle\ell_{n^{\prime}}\right\rangle(\Omega)$, defined by

$$
\begin{gathered}
P_{\bar{\Omega}}(\Omega)=\frac{N_{c}(\Omega)}{N_{c}}, \\
\left\langle f_{\bar{n}}\right\rangle(\Omega)=\frac{1}{N_{c}(\Omega)} \sum_{c \in \mathcal{S}(\Omega)} f_{\bar{n}}^{c}, \\
\left\langle f_{\bar{t}}\right\rangle(\Omega)=\frac{1}{N_{c}(\Omega)} \sum_{c \in \mathcal{S}(\Omega)} f_{\bar{t}}^{c}, \\
\left\langle\ell_{\bar{n}}\right\rangle(\Omega)=\frac{1}{N_{c}(\Omega)} \sum_{c \in \mathcal{S}(\Omega)} \ell_{\bar{n}}^{c}, \\
\left\langle\ell_{t}\right\rangle(\Omega)=\frac{1}{N_{c}(\Omega)} \sum_{c \in \mathcal{S}(\Omega)} \ell_{t}^{c},
\end{gathered}
$$

where $N_{c}$ is the total number of contacts, $N_{c}(\Omega)$ the number of contacts pointing in the direction $\Omega,(\bar{n}, \bar{t})=(n, t)$ if $\bar{\Omega}=\Omega$, and $(\bar{n}, \bar{t})=\left(n^{\prime}, t^{\prime}\right)$ if $\bar{\Omega}=\Omega^{\prime}$.

Under axisymmetric conditions, these probability density functions are independent of the azimuthal angle $\phi$. Figure 14 displays a polar representation of these functions in the $\theta$ plane in the residual state for $S 6$. We observe an anisotropic behavior 
in all cases. The peak value occurs along the compression axis $\theta=0$ for $P_{\bar{\theta}}(\theta)$ and for $\left\langle f_{n}\right\rangle(\theta)$, where $\bar{\theta}$ stands for $\theta$ or $\theta^{\prime}$. The peak values for $\left\langle\ell_{t}\right\rangle(\theta),\left\langle f_{t}\right\rangle(\theta)$, and $\left\langle f_{t^{\prime}}\right\rangle(\theta)$ occur at $\theta=\pi / 4$. For $\left\langle\ell_{n}\right\rangle(\theta)$ and $\left\langle\ell_{n^{\prime}}\right\rangle(\theta)$ the probability density function's are nearly isotropic. We see also that $\left\langle f_{n}\right\rangle(\theta) \simeq\left\langle f_{n^{\prime}}\right\rangle(\theta)$.

The simple shapes of the above functions suggest that an approximation based on spherical harmonics at leading terms should capture their anisotropic behavior. There are nine second-order basis functions $Y_{m}^{l}(\theta, \phi)$ but only the functions compatible with the symmetries of the problem (i.e., independent with respect to $\phi$ and $\pi$ periodic in $\theta$ ) are admissible. The only admissible functions are, therefore, $Y_{0}^{0}=1$ and $Y_{2}^{0}=3 \cos ^{2} \theta-1$. Hence, within the harmonic model of fabric and force, we have [24,56]

$$
\left\{\begin{array}{l}
P_{\bar{\theta}}(\theta)=\frac{1}{4 \pi}\left\{1+\bar{a}_{c}\left[3 \cos ^{2} \theta-1\right]\right\} \\
\left\langle\ell_{\bar{n}}\right\rangle(\theta)=\left\langle\ell_{\bar{n}}\right\rangle\left\{1+a_{l \bar{n}}\left[3 \cos ^{2} \theta-1\right]\right\} \\
\left\langle\ell_{t}\right\rangle(\theta)=\left\langle\ell_{n}\right\rangle a_{l t} \sin 2 \theta \\
\left\langle f_{\bar{n}}\right\rangle(\theta)=\left\langle f_{\bar{n}}\right\rangle\left\{1+a_{f \bar{n}}\left[3 \cos ^{2} \theta-1\right]\right\} \\
\left\langle f_{\bar{t}}\right\rangle(\theta)=\left\langle f_{\bar{n}}\right\rangle a_{f \bar{t}} \sin 2 \theta
\end{array}\right.
$$

where $\left\langle\ell_{\bar{n}}\right\rangle$ and $\left\langle f_{\bar{n}}\right\rangle$ are the mean normal and radial lengths and the mean normal and radial forces. The anisotropy parameters are $\left(a_{c}, a_{l n}, a_{f n}, a_{f t}, a_{l t}\right)$ in the frame $(\boldsymbol{n}, \boldsymbol{t}, \boldsymbol{s})$, and $\left(a_{c}^{\prime}, a_{l n^{\prime}}, a_{f n^{\prime}}, a_{f t^{\prime}}\right)$ in the frame $\left(\boldsymbol{n}^{\prime}, \boldsymbol{t}^{\prime}, \boldsymbol{s}^{\prime}\right)$. As a result of axial symmetry, the privileged direction of all these functions is the vertical direction $(\theta=0)$.

In the following, we refer to $a_{c}$ as contact anisotropy, to $a_{c}^{\prime}$ as branch orientation anisotropy, to $\left(a_{l \bar{n}}, a_{l t}\right)$ as branch length anisotropies, and to $\left(a_{f \bar{n}}, a_{f \bar{t}}\right)$ as normal and tangential or radial and orthoradial force anisotropies, respectively, depending on the local frame used for harmonic decomposition. In practice, the values of all anisotropy parameters can be calculated from generalized fabric tensors presented in Appendix A.

Using the harmonic approximation (26), the averages given by Eqs. (19) and (20) can be evaluated by integration with respect to space directions $\theta$. Neglecting cross products between all anisotropies, we get the following simple relations [8,24,56,57]:

$$
\frac{q}{p} \simeq\left\{\begin{array}{l}
\frac{2}{5}\left(a_{c}+a_{l n}+a_{l t}+a_{f n}+a_{f t}\right), \\
\frac{2}{5}\left(a_{c}^{\prime}+a_{l n^{\prime}}+a_{f n^{\prime}}+a_{f t^{\prime}}\right)
\end{array}\right.
$$

These equations express the normalized shear stress as a function of the anisotropy parameters with two different local frames. These equations hold only under axial symmetry. In the most general case, more anisotropy parameters should be introduced.

\section{Granular texture}

Here, we analyze the anisotropies described in the previous section and their respective roles in the shear strength as a function particle angularities. Figure 15 displays the variations of the orientation anisotropies of contacts and branch vectors, respectively $a_{c}$ and $a_{c}^{\prime}$, averaged in the steady state as a function of $\langle\alpha\rangle$. We see that $a_{c}$ increases with $\langle\alpha\rangle$ up to a maximum value and then declines for $\langle\alpha\rangle>0.6$. $a_{c}^{\prime}$ follows a similar trend with an increasing phase followed by a decreasing phase for $\langle\alpha\rangle>0.2$. It is often admitted that the shear strength in granular media is a consequence of the buildup of an anisotropic structure. However, here we have a nonmonotonic

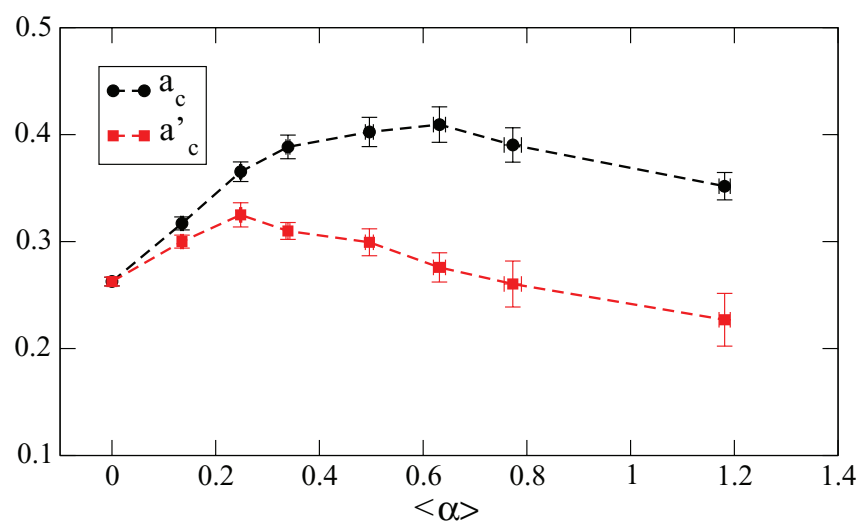

FIG. 15. (Color online) Contact anisotropy $a_{c}$ and branch vector anisotropy $a_{c}^{\prime}$ as a function of angularity $\langle\alpha\rangle$ averaged in the residual state. Error bars represent the standard deviation in the residual state.

evolution of both anisotropies as a function of $\langle\alpha\rangle$ although the shear strength continues to increase.

Figure 16 shows the variation of branch-vector length anisotropies $a_{l n}, a_{l t}$, and $a_{l n^{\prime}}$ averaged in the steady state as a function of $\langle\alpha\rangle$. These parameters are negligibly small (in particular, $a_{l n}=a_{l n^{\prime}} \simeq 0$ ) for all values of $\langle\alpha\rangle$. This is due to the absence of shape eccentricity of the particles and also because of the low span in the particle size distribution [58]. It is remarkable also that $a_{l t}$ is negative and increases in absolute value in the residual state. This means that the particles tend to form longer branch vectors with their neighbors in the direction of extension rather than compression [8].

\section{Force transmission and friction mobilization}

The normal and tangential force anisotropies $a_{f n}$ and $a_{f t}$ are plotted in Fig. 17 as a function of $\langle\alpha\rangle$, together with the radial and orthoradial force anisotropies $a_{f n^{\prime}}$ and $a_{f t^{\prime}}$ averaged in the steady state. We see that $a_{f n} \simeq a_{f n^{\prime}}$. In connection with the variations of $a_{c}$ and $a_{c}^{\prime}$, the increase of $a_{f n}$ and $a_{f n^{\prime}}$ shows that stronger force chains are transmitted along the principal stress direction and thus the mean normal and radial forces increase, too. It is worth mentioning that $a_{f n}$

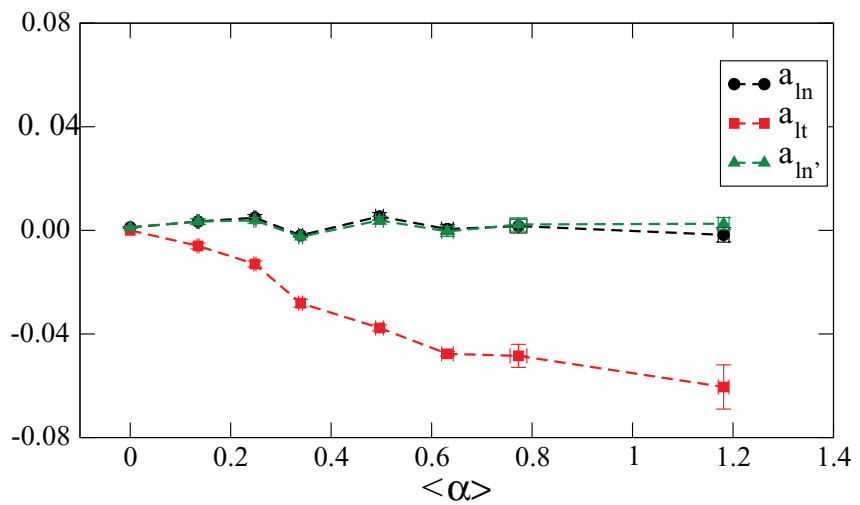

FIG. 16. (Color online) Normal and tangential branch-vector length anisotropies $a_{l n}, a_{l t}$, and $a_{l n^{\prime}}$ as a function of angularity $\langle\alpha\rangle$ averaged in residual state. Error bars represent the standard deviation in the residual state. 


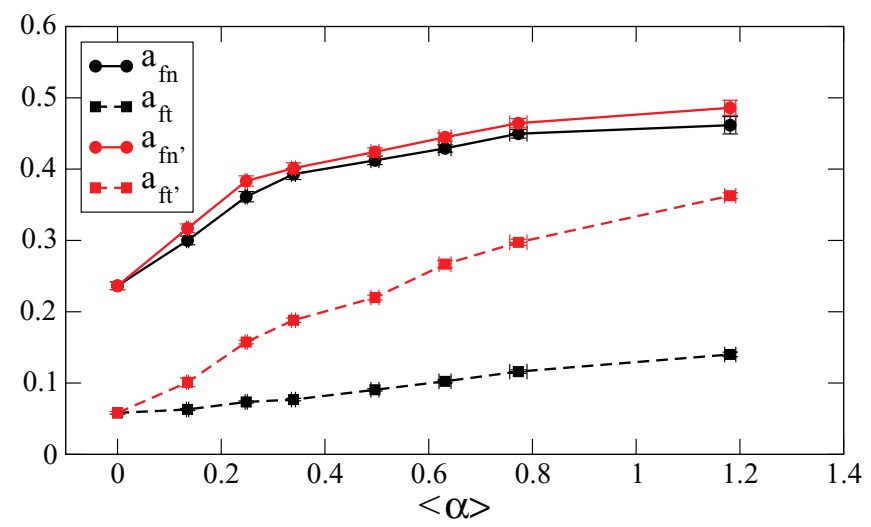

FIG. 17. (Color online) Normal and tangential force anisotropies $a_{n}$ and $a_{t}$, and radial and orthoradial force anisotropies $a_{n}^{\prime}$ and $a_{t}^{\prime}$, as a function of $\langle\alpha\rangle$ in the residual state. The error bars represent the standard deviation in the residual state.

and $a_{f n^{\prime}}$ increase slowly at higher angularity while $a_{c}$ and $a_{c}^{\prime}$ decrease at the same time. This means that the stress along the compression axis is sustained by less contacts but stronger forces. As mentioned previously, the new contacts coming into play at higher angularity are mainly face-face contacts, which capture stronger forces.

Figure 17 shows also the variation of tangential and orthoradial force anisotropies. We see that both $a_{f t}$ and $a_{f t^{\prime}}$ increase with $\langle\alpha\rangle$. In particular, $a_{f t^{\prime}}$ is larger than $a_{f t}$ and increases also faster. The dry friction law implies that $\left|f_{\bar{t}}\right|<\bar{\mu} f_{\bar{n}}$, where $\bar{\mu}=\mu$ in the contact frame and $\bar{\mu}=$ $\left(f_{n^{\prime}} / f_{n}\right) \sqrt{\left(1+\mu^{2}\right)-\left(f_{n} / f_{n^{\prime}}\right)^{2}}$ in the branch-vector frame. Hence, the ratio $\bar{I}_{c}=\left|f_{\bar{t}}\right| /\left(\bar{\mu} f_{\bar{n}}\right)$ can be used as an index of friction mobilization for a contact $c$. A map of mobilized friction forces is shown in Fig. 18 for S5. At the scale of the packing, the index $I_{\bar{\mu}}=\left\langle\left|f_{\bar{t}}\right|\right\rangle /\left\langle\bar{\mu} f_{\bar{n}}\right\rangle$ represents the mean friction mobilization. Integrating Eq. (26)(e) in the range of $[0, \pi / 2]$ and assuming that $\bar{\mu}$ and $f_{n^{\prime}}$ are weakly correlated, we get

$$
I_{\bar{\mu}}=\frac{5}{2 \bar{\mu}} a_{\bar{t}} .
$$

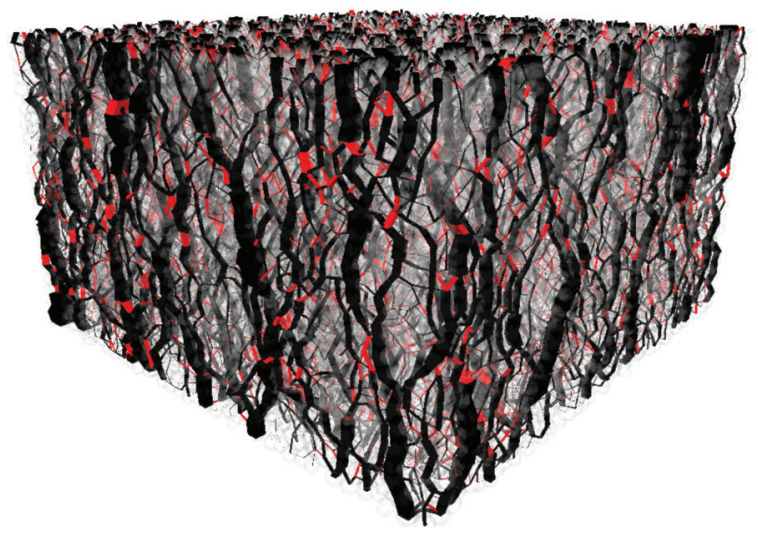

FIG. 18. (Color online) Snapshot of radial forces for the sample $S 5$. Line thickness is proportional to the radial force. Mobilized forces $\left(I_{c} \simeq 1\right)$ are in red.

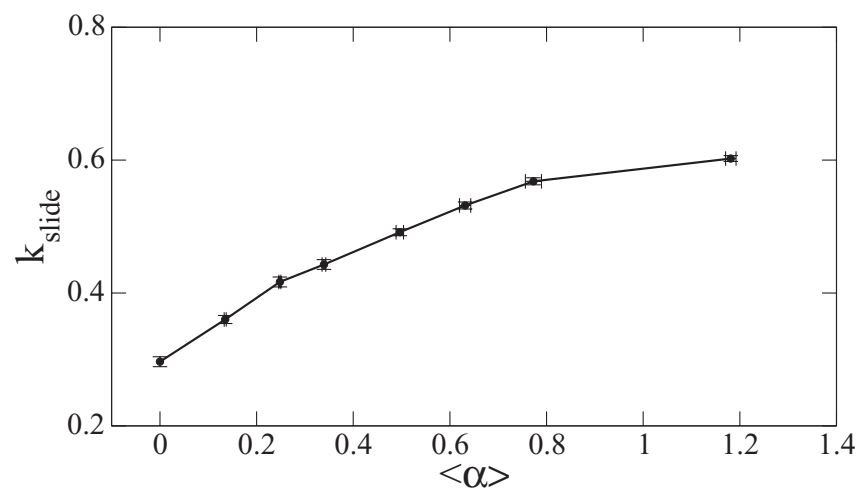

FIG. 19. Proportion of sliding contacts as a function of $\langle\alpha\rangle$ averaged in the residual state. The error bars represent the standard deviation.

As a result, the increase of $a_{f t}$ and $a_{f t^{\prime}}$ with $\langle\alpha\rangle$ implies that at higher angularity the force balance is ensured by a strong activation of friction forces. This effect can also be evidenced by plotting the proportion $k_{\text {slide }}$ of sliding contacts as a function of $\langle\alpha\rangle$; see Fig. 19. As expected, $k_{\text {slide }}$ increases as the particles become more and more angular, reaching values as high as 0.6,i.e., 2 times above those measured in the packing composed of spheres. This finding can be related to a recent work showing that one of the main influencing factors behind the mechanical behavior of granular systems composed of angular particles is the partial hindrance of rotations pushing the particles to slide over one another to accommodate the imposed deformations [59]. The contributions of each contact type to the force anisotropies are analyzed more precisely in the following section.

The anisotropy parameters can also be calculated at the stress peak state. They are not shown here, but the trends are similar, except for $a_{f n}^{+}$and $a_{f n^{\prime}}^{+}$, which decline at higher values of $\langle\alpha\rangle$. Figure 20 displays the peak-state and residual-state values of the normalized shear stresses $(q / p)^{+}$and $(q / p)^{*}$, respectively, as a function of $\langle\alpha\rangle$ calculated both directly from the simulation data and from Eqs. (27a) and (27b) separately for the contact frames and branch-vector frames. We see that Eq. (27b), which is based on general considerations, expressed

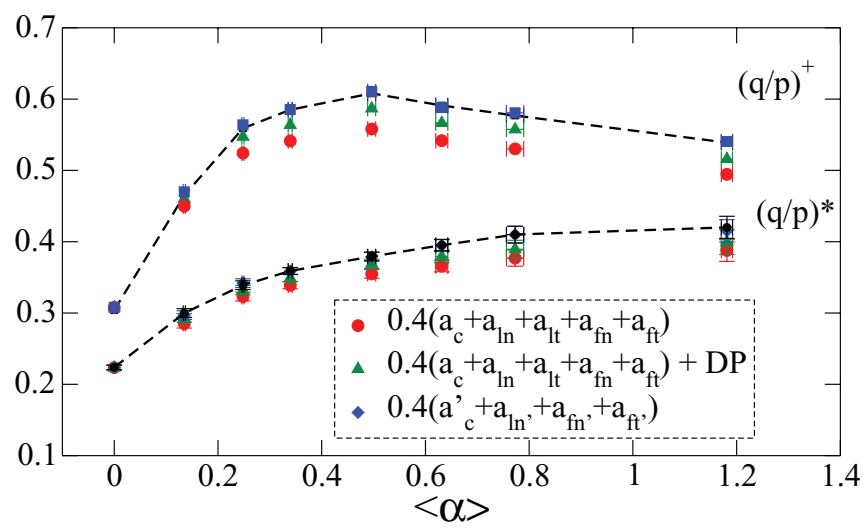

FIG. 20. (Color online) Normalized shear stress $(q / p)^{+}$and $(q / p)^{*}$ at peak and residual states as a function of $\langle\alpha\rangle$ together with the analytical expressions given by Eqs. (27a), (27b), and (B1). The error bars represent the standard deviation in the residual state. 


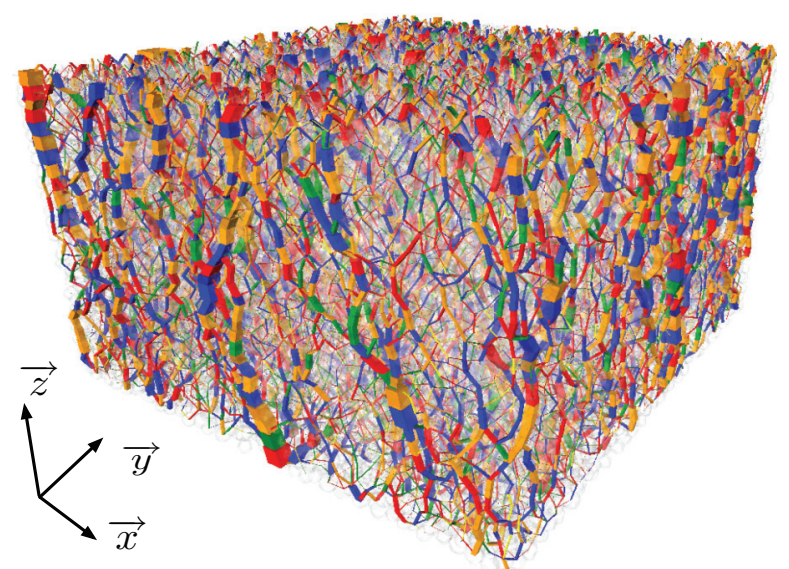

FIG. 21. (Color online) Snapshot of radial forces for S5. Line thickness is proportional to the radial force. $e e$ contacts are in green, $f v$ contacts in red, $f e$ contacts in blue, and $f f$ contacts in yellow.

in the branch-vector frame, provides an excellent fit, whereas the approximation proposed in the contact frame is correct but it underestimates the shear strength both at peak and residual states. This can, however, be easily corrected by including the cross products among the anisotropies, which are neglected [56]; see Appendix B .

Equations (27a) and (27b) allows us to evaluate the relative weights of fabric anisotropy, force chains, and friction mobilization with regard to shear strength. We see that, expressed in the contact frame, the increase of the shear strength at low angularities is due to an increase of both contact and force anisotropies, whereas the saturation trend is a consequence of a rapid falloff of the contact anisotropies compensated by an increase of the tangential force anisotropy. Expressed in the branch-vector frame, the variation of the shear strength follows that of the radial force anisotropy. In other words, the loss of branch-vector anisotropy is fully compensated by the gain in radial force anisotropy.

\section{Role of contact types}

In this section, we would like to quantify the relative contributions of each type of contact to the shear strength and anisotropy parameters. A map of contact forces projected along the branch vectors is displayed in Fig. 21 for $S 5$ in different colors according to the contact type. The network of strong force chains appears to be mostly composed of $f f$ and $f e$ contacts mediated occasionally by $e e$ and $f v$ contacts.

Let us consider the discrete expression of the stress tensor in Eq. (3) and partition it as a sum of different terms, each containing only the contribution of all contacts of the same type,

$$
\sigma=\sigma_{f f}+\sigma_{f e}+\sigma_{f v}+\sigma_{e e},
$$

where $\sigma_{f f}, \sigma_{f e}, \sigma_{f v}$, and $\sigma_{e e}$ are obtained by restricting the summation to $f f, f e, f v$, and $e e$ contacts, respectively. The respective stress deviators $q_{f f}, q_{f e}, q_{f v}$, and $q_{e e}$ normalized by the pression $p$ are shown in Fig. 22. We see that, at low angularity $q_{e e} / p \sim q_{f e} / p<q_{f f} / p$ even if $k_{f e}<k_{f f}<k_{e e}$. At higher angularities, the stress plateau appears to be a consequence of a decrease of $q_{f f} / p$ (resulting from the

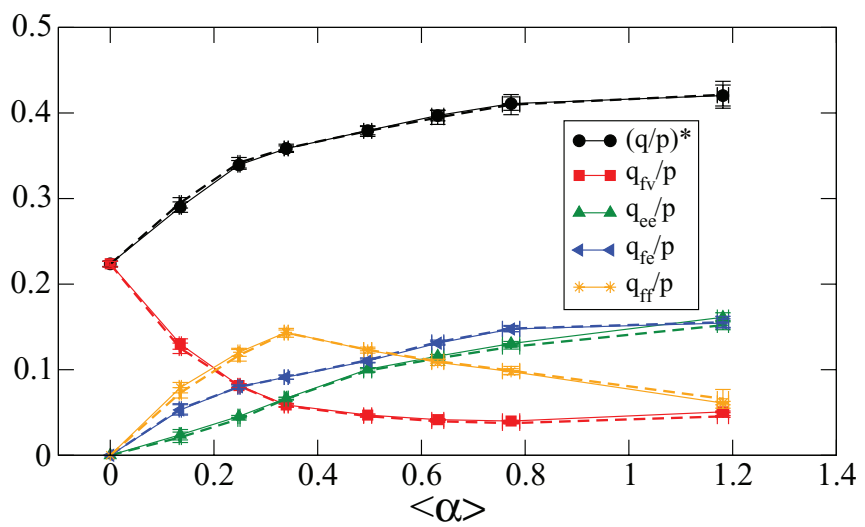

FIG. 22. (Color online) Shear strength $(q / p)^{*}$ for $f v, e e, f e$, and $f f$ contacts as a function $\langle\alpha\rangle$, together with the harmonic approximation fits in $\left(\boldsymbol{n}^{\prime}, \boldsymbol{t}^{\prime}\right)$ frame $(\cdots)$. The error bars represent the standard deviation in the residual state.

decrease of $k_{f f}$ ) compensated by an increase of both $q_{f e} / p$ and $q_{e e} / p$. It is remarkable that $q_{f e} / p \sim q_{e e} / p$, whereas $k_{f e} \ll k_{e e}$. We also see that $q_{f v} / p$ decreases with $\langle\alpha\rangle$ as a result of the decrease of $k_{f v}$. In this way, the growth of the number of $f f$ and $f e$ contacts shown in Fig. 11 is clearly the main origin of the increase of shear strength at low angularity.

We now consider the "relative" texture and force anisotropies supported by each contact type. Since the texture and force anisotropies expressed in the branch frames are simpler and more accurate than in the contact frames, we restrict here our analysis to the branch-vector partition. By partitioning the fabric and force tensors mentioned in Appendix A as we did for the stress tensor, we can also calculate the partial fabric and force anisotropies $\left(a_{c \gamma}^{\prime}, a_{l n^{\prime} \gamma}, a_{f n^{\prime} \gamma}\right.$, and $\left.a_{f t^{\prime} \gamma}\right)$, where $\gamma$ stands alternatively for $\{f f, f s, f v, e e\}$. In our case, assuming that in the steady state the principal directions of the partial angular functions of branch vectors and forces are the same as the global branch and force angular functions, we have

$$
\frac{q_{\gamma}}{p} \simeq \frac{2}{5}\left(a_{c \gamma}^{\prime}+a_{l n^{\prime} \gamma}+a_{f n^{\prime} \gamma}+a_{f t^{\prime} \gamma}\right),
$$

As illustrated in Fig. 22, this relation holds for each contact type.

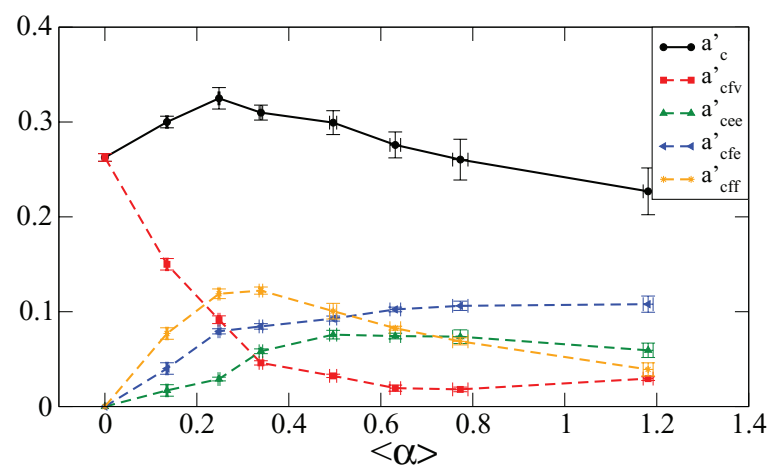

FIG. 23. (Color online) Contact anisotropy in the frame $\left(\boldsymbol{n}^{\prime}, \boldsymbol{t}^{\prime}\right)$ for $f v, e e, f e$, and $f f$ contacts as a function $\langle\alpha\rangle$. The error bars represent the standard deviation in the residual state. 

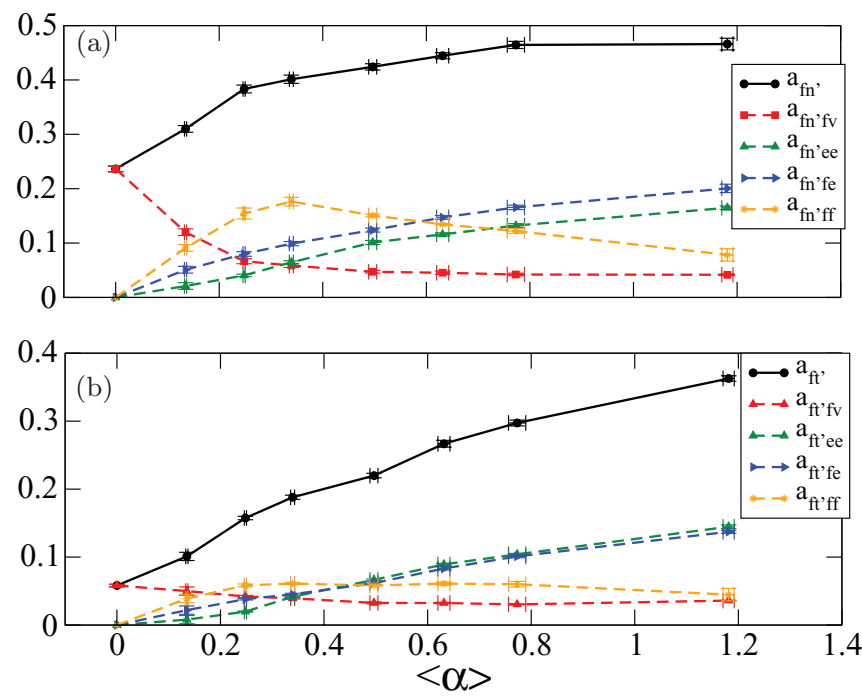

FIG. 24. (Color online) Radial (a) and orthoradial (b) force anisotropy in the $\left(\boldsymbol{n}^{\prime}, \boldsymbol{t}^{\prime}\right)$ frame for $f v, e e, f e$, and $f f$ contacts as a function $\langle\alpha\rangle$. The error bars represent the standard deviation in the residual state.

Figures 23 and 24 display the variation of $a_{c \gamma}^{\prime}, a_{f n^{\prime} \gamma}$, and $a_{f t^{\prime} \gamma}$ averaged in the steady state as a function of $\langle\alpha\rangle$. The decrease of $a_{c}^{\prime}$ is a consequence of the decrease of $a_{c f f}^{\prime}$, whereas $a_{c e e}^{\prime}\left(<a_{c f v}^{\prime}\right)$ remains nearly constant with $\langle\alpha\rangle$. This is clearly due to the decrease of $k_{f f}$. In the same way, the saturation of $a_{f n^{\prime}}$ results from the decrease of $a_{f n^{\prime} f f}$ compensated by the increase of both $a_{f n^{\prime} e e}$ and $a_{f n^{\prime} f s}$. Finally, the increase of $a_{f t^{\prime}}$ is attributed to the increase of both $a_{f t^{\prime} e e}$ and $a_{f t^{\prime} f s}$. In particular, it is remarkable that $a_{f t^{\prime} e e}=a_{f t^{\prime} f s}$ and $a_{f t^{\prime} f f}$ is constant, whereas $k_{e e}>k_{f e}$ and $k_{f f}$ decreases with $\langle\alpha\rangle$. In other words, even if the proportion of contacts involving a face is as small as $f f$ contacts or less numerous than $e e$ contacts, this population of contacts involves a higher mobilization of friction.

\section{CONCLUSIONS}

In this paper, a systematic analysis of the effect of angular shape on the quasistatic rheology of sheared granular materials was carried out by means of numerical simulations. Angular particle shapes were modeled as irregular polyhedral particles in the framework of the CD method. This method is particularly relevant to address the quasistatic plastic deformations of dense and large systems by avoiding numerical artifacts arising from a finite stiffness at the contacts between flat faces and edges of the particles. The particles are irregular polyhedra generated from spheres and characterized by a single angularity parameter $\langle\alpha\rangle$, defined as the mean exterior angle between adjacent faces. The angularity was gradually increased from $\alpha=0$ (spheres) to $\langle\alpha\rangle \sim 1.18$ (irregular octahedron). The rheological behavior of several packings of 40000 particles subjected to triaxial compression were analyzed as a function of $\langle\alpha\rangle$.

An important finding of this work is that the shear strength first increases with the angularity but saturates as the particles become more angular. Unexpectedly, very small deviations from spherical shape have stronger effect on the shear strength than large variations of angularity at low number of faces. To understand the microscopic origins of this behavior, we performed an additive partition of the stress tensor based on a harmonic approximation of the angular dependence of the average local forces and branch vectors expressed either in the contact frame or in the branch-vector frame. These two descriptions provide complementary points of view of the microstructure. As a result of the aspherical shape of the particles, the contact network is different from the network of neighboring particles, materialized by the branch vectors, and one important issue is then how the particle shape is reflected in the differences between the two viewpoints. The nature of the contact network is obviously guided by the organization of the contact types. In contrast, the branch network reflects mainly the spatial distribution of the particles. In this sense, this network is may be used as the reference microstructure to characterize the contact network.

Our detailed analysis of the corresponding fabric and force anisotropies allowed us to highlight the microscopic mechanisms that lead to the dependence of shear strength with respect to $\langle\alpha\rangle$. The increase of shear strength results from that of both contact and force anisotropies. The plateau of the shear strength is a consequence of the decrease of the contact anisotropy compensated by the increase of the tangential anisotropy. This means that the increasing mobilization of friction forces is the key effect of particle angularity. In particular, the proportion of sliding contacts increases strongly as the particles become more angular.

In this investigation, the sphericity of the particles was fixed to $\sim 1$ in order to isolate the effect of particle angularity. However, it should be interesting to vary the sphericity of the particles in order to compare the effects of flatness or elongation of the particles with those of angularity. In the same way, since friction mobilization plays a major role at large angularity, it should be interesting to investigate also the influence of the coefficient of friction for angular particles.

\section{ACKNOWLEDGMENT}

We thank Nicolas Estrada from Los Andes University for fruitful discussions.

\section{APPENDIX A: FABRIC TENSORS}

It is convenient to estimate the anisotropy parameters from the following fabric and force tensors [8,60]:

$$
\begin{aligned}
\bar{F}_{\alpha \beta} & =\int_{\Omega} \bar{n}_{\alpha} \bar{n}_{\beta} P_{\bar{\Omega}}(\Omega) d \Omega, \\
\chi_{\alpha \beta}^{l \bar{n}} & =\frac{1}{\left\langle\ell_{\bar{n}}\right\rangle} \int_{\Omega}\left\langle\ell_{\bar{n}}\right\rangle(\Omega) \bar{n}_{\alpha} \bar{n}_{\beta} P_{\bar{\Omega}}(\Omega) d \Omega, \\
\chi_{\alpha \beta}^{l \bar{t}} & =\frac{1}{\left\langle\ell_{\bar{n}}\right\rangle} \int_{\Omega}\left\langle\ell_{\bar{t}}\right\rangle(\Omega) \bar{n}_{\alpha} \bar{t}_{\beta} P_{\bar{\Omega}}(\Omega) d \Omega, \\
\chi_{\alpha \beta}^{f \bar{n}} & =\frac{1}{\left\langle f_{\bar{n}}\right\rangle} \int_{\Omega}\left\langle f_{\bar{n}}\right\rangle(\Omega) \bar{n}_{\alpha} \bar{n}_{\beta} P_{\bar{\Omega}}(\Omega) d \Omega, \\
\chi_{\alpha \beta}^{f \bar{t}} & =\frac{1}{\left\langle f_{\bar{n}}\right\rangle} \int_{\Omega}\left\langle f_{\bar{t}}\right\rangle(\Omega) \bar{n}_{\alpha} \bar{t}_{\beta} P_{\bar{\Omega}}(\Omega) d \Omega,
\end{aligned}
$$


where $\alpha$ and $\beta$ design the components in the considered frame. Note that, by construction, we have $\chi_{\alpha \beta}^{l t^{\prime}}=0$. From Eqs. (26) and (A1), assuming that under axial symmetry the major principal direction of all tensors is vertical $(\theta=0)$, the following relations are easily obtained:

$$
\begin{aligned}
\bar{a}_{c} & =\frac{5}{2}\left(\bar{F}_{1}-\bar{F}_{3}\right) /\left(\bar{F}_{1}+\bar{F}_{2}+\bar{F}_{3}\right), \\
a_{l \bar{n}} & =\frac{5}{2}\left(\chi_{1}^{l \bar{n}}-\chi_{3}^{l \bar{n}}\right) /\left(\chi_{1}^{l \bar{n}}+\chi_{2}^{l \bar{n}}+\chi_{3}^{l \bar{n}}\right)-\bar{a}_{c}, \\
a_{l \bar{t}} & =\frac{5}{2}\left(\chi_{1}^{\bar{l}}-\chi_{3}^{\bar{l}}\right) /\left(\chi_{1}^{\bar{l}}+\chi_{2}^{\bar{l}}+\chi_{3}^{\bar{l}}\right)-\bar{a}_{c}-a_{l \bar{n}}, \\
a_{f \bar{n}} & =\frac{5}{2}\left(\chi_{1}^{f \bar{n}}-\chi_{3}^{f \bar{n}}\right) /\left(\chi_{1}^{f \bar{n}}+\chi_{2}^{f \bar{n}}+\chi_{3}^{f \bar{n}}\right)-\bar{a}_{c}, \\
a_{f \bar{t}} & =\frac{5}{2}\left(\chi_{1}^{\bar{f}}-\chi_{3}^{\bar{f}}\right) /\left(\chi_{1}^{\bar{f}}+\chi_{2}^{\bar{f}}+\chi_{3}^{\bar{f}}\right)-\bar{a}_{c}-a_{f \bar{n}},
\end{aligned}
$$

where $\chi^{\bar{l}}=\chi^{l \bar{n}}+\chi^{l \bar{t}}, \chi^{\bar{f}}=\chi^{f \bar{n}}+\chi^{f \bar{t}}$ and the indices 1 , 2 , and 3 refer to the principal values of each tensor. By construction, we have $\left(\bar{F}_{1}+\bar{F}_{2}+\bar{F}_{3}\right)=1,\left(\chi_{1}^{\bar{l}}+\chi_{2}^{\bar{l}}+\chi_{3}^{\bar{l}}\right)=$ 1 , and $\left(\chi_{1}^{\bar{f}}+\chi_{2}^{\bar{f}}+\chi_{3}^{\bar{f}}\right)=1$. Note that $\bar{a}_{c}, a_{f \bar{n}}$, and $a_{f \bar{t}}$ are always positive, whereas $\bar{a}_{l n}$ and $a_{l \bar{t}}$ may be negative.

\section{APPENDIX B: SHEAR STRENGTH APPROXIMATION AT SECOND ORDER}

The shear strength can be expanded to the second order without neglecting the cross products between anisotropies. In the contact frame we have [56]

$$
\begin{aligned}
\frac{q}{p} \simeq & \frac{2}{5}\left(a_{c}+a_{l n}+a_{l t}+a_{f n}+a_{f t}\right) \\
& +\frac{4}{105}\left(a_{c} \cdot a_{f n}+a_{c} \cdot a_{l n}+a_{l n} \cdot a_{f n}\right) \\
& +\frac{16}{105}\left(a_{c} \cdot a_{f t}+a_{c} \cdot a_{l t}+a_{l n} \cdot a_{f t}+a_{l t} \cdot a_{f n}\right) .
\end{aligned}
$$

As we see in Fig. 20, Eq. (B1) gives a better approximation than Eq. (27a). Note also that the excellent fits to the data by Eqs. (27b) and ((B1) indicate that the assumption of weak correlations between forces and branch vector lengths is indeed a good approximation. This can be checked directly from the simulation data by evaluating the Pearson correlation parameter $C_{\ell f}$ defined by

$$
C_{\ell f}=\frac{\langle\ell f\rangle}{\sqrt{\left\langle\ell^{2}\right\rangle} \sqrt{\left\langle f^{2}\right\rangle}},
$$

where $f$ is the magnitude of the contact force. We find that $\left|C_{\ell f}\right|$ does not exceed 0.05 .
[1] J. Mitchell and K. Soga, Fundamentals of Soil Behavior (Wiley, New York, 2005).

[2] Edited by J. Santamarina, Soils and Waves (Wiley \& Sons, New York, 2009).

[3] Cegeo, B. Saint-Cyr, K. Szarf, C. Voivret, E. Azéma, V. Richefeu, J.-Y. Delenne, G. Combe, C. Nouguier-Lehon, P. Villard et al., Europhys. Lett. 98, 44008 (2012).

[4] A. Donev, F. H. Stillinger, P. M. Chaikin, and S. Torquato, Phys. Rev. Lett. 92, 255506 (2004).

[5] A. Donev, I. Cisse, D. Sachs, E. Variano, F. Stillinger, R. Connelly, S. Torquato, and P. Chaikin, Science 303, 990 (2004).

[6] W. Man, A. Donev, F. H. Stillinger, M. T. Sullivan, W. B. Russel, D. Heeger, S. Inati, S. Torquato, and P. M. Chaikin, Phys. Rev. Lett. 94, 198001 (2005).

[7] S. Sacanna, L. Rossi, A. Wouterse, and A. P. Philipse, J. Phys.: Condens. Matter 19, 376108 (2007).

[8] E. Azéma and F. Radjaï, Phys. Rev. E 81, 051304 (2010).

[9] C. Nouguier-Lehon, C. R. Méc. 338, 587 (2010).

[10] I. Zuriguel and T. Mullin, Proc. R. Soc. A 464, 99 (2008).

[11] R. C. Hidalgo, I. Zuriguel, D. Maza, and I. Pagonabarraga, Phys. Rev. Lett. 103, 118001 (2009).

[12] T. Kanzaki, M. Acevedo, I. Zuriguel, I. Pagonabarraga, D. Maza, and R. Hidalgo, Eur. Phys. J. E 34, 133 (2011).

[13] T. Borzsonyi, B. Szabo, S. Wegner, K. Harth, J. Torok, E. Somfai, T. Bien, and R. Stannarius, Phys. Rev. E 86, 051304 (2012).

[14] T. Börzsönyi, B. Szabó, G. Törös, S. Wegner, J. Török, E. Somfai, T. Bien, and R. Stannarius, Phys. Rev. Lett. 108, 228302 (2012).

[15] M. Acevedo, R. C. Hidalgo, I. Zuriguel, D. Maza, and I. Pagonabarraga, Phys. Rev. E 87, 012202 (2013).
[16] K. Szarf, G. Combe, and P. Villard, Powder Technol. 208, 279 (2011).

[17] B. Saint-Cyr, J.-Y. Delenne, C. Voivret, F. Radjai, and P. Sornay, Phys. Rev. E 84, 041302 (2011).

[18] E.-G. Nezami, Y. Hashash, D. Zaho, and J. Ghaboussi, Comput. Geotech. 31, 575 (2004).

[19] F. Alonso-Marroquin and H. J. Herrmann, Phys. Rev. E 66, 021301 (2002).

[20] G. Saussine, C. Cholet, P. Gautier, F. Dubois, C. Bohatier, and J. Moreau, Comput. Methods Appl. Mech. Eng. 195, 2841 (2006).

[21] E. Azéma, F. Radjai, R. Peyroux, F. Dubois, and G. Saussine, Phys. Rev. E 74, 031302 (2006).

[22] E. Azéma, F. Radjai, R. Peyroux, and G. Saussine, Phys. Rev. E 76, 011301 (2007).

[23] E. Azéma, F. Radjai, R. Peyroux, V. Richefeu, and G. Saussine, Eur. Phys. J. E 26, 327 (2008).

[24] E. Azéma, F. Radjai, and G. Saussine, Mech. Mater. 41, 729 (2009).

[25] S. A. Galindo-Torres and D. M. Pedroso, Phys. Rev. E 81, 061303 (2010).

[26] S. Mack, P. Langston, C. Webb, and T. York, Powder Technol. 214, 431 (2011).

[27] F. Radjai and F. Dubois (eds.), Discrete Numerical Modeling of Granular Materials (Wiley-ISTE, New York, 2011).

[28] E. Azema, Y. Descantes, N. Roquet, J.-N. Roux, and F. Chevoir, Phys. Rev. E 86, 031303 (2012).

[29] S. J. Lee, Y. M. Hashash, and E. G. Nezami, Comput. Geotech. 43, 92 (2012).

[30] S. Torquato and Y. Jiao, Phys. Rev. E 80, 041104 (2009).

[31] K. C. Smith, M. Alam, and T. S. Fisher, Phys. Rev. E 82, 051304 (2010). 
[32] J. Baker and A. Kudrolli, Phys. Rev. E 82, 061304 (2010).

[33] E. Azema, N. Estrada, and F. Radjai, Phys. Rev. E 86, 041301 (2012).

[34] J. J. Moreau, in Powders \& Grains 93 (A. A. Balkema, Rotterdam, 1993), p. 227.

[35] M. Jean, Comput. Methods Appl. Mech. Eng. 177, 235 (1999).

[36] F. Radjaï and E. Azéma, Eur. J. Env. Civil Eng. 13, 204 (2009).

[37] H. Wadell, J. Geol. 43, 250 (1935).

[38] F. Dubois, in [27], Chapter on Numerical Modeling of Granular Media Composed of Polyhedral Particles.

[39] P. A. Cundall, Int. J. Rock Mech. Min. Sci. Geomech. Abstr. 25, 107 (1988).

[40] E. Nezami, Y. Hashash, D. Zaho, and J. Ghaboussi, Int. J. Numer. Anal. Methods Geomech. 30, 783 (2006).

[41] S.-W. Chang and C.-S. Chen, Int. J. Numer. Methods Eng. 74, 734 (2008).

[42] G. Saussine, Ph.D. thesis, Université Montpellier II, 2004.

[43] F. Dubois and M. Jean, LMGC90 une plateforme de développement dédiée à la modélisation des problèmes d'interaction, in 6ème Colloque National en Calcul des Structures, edited by M. Potier-Ferry, M. Bonnet, and A. Bignonnet, Giens (2003), Vol. 1, pp. 111-118 (unpublished).

[44] M. Renouf and P. Alart, Comput. Methods Appl. Mech. Eng. 194, 2019 (2005).

[45] F. Dubois and M. Jean, in Analysis and Simulation of Contact Problems, edited by P. Wriggers and U. Nackenhorst, Lecture Notes in Applied and Computational Mechanics Vol. 27 (Springer, Berlin/Heidelberg, 2006), pp. 375-378.
[46] V. Visseq, A. Martin, D. Iceta, E. Azema, D. Dureisseix, and P. Alart, Comput. Mech. 49, 709 (2012).

[47] C. Voivret, F. Radjaï, J.-Y. Delenne, and M. S. El Youssoufi, Phys. Rev. E 76, 021301 (2007).

[48] GDR-MiDi, Eur. Phys. J. E 14, 341 (2004).

[49] J. J. Moreau, in Friction, Arching, Contact Dynamics, edited by D. E. Wolf and P. Grassberger (World Scientific, Singapore, 1997), pp. 233-247.

[50] A. Schofield and C. Wroth, Critical State of Soil Mechanics (McGraw Hill, New York, 1968).

[51] M. Boton, E. Azéma, N. Estrada, F. Radjai, and A. Lizcano, Phys. Rev. E 87, 032206 (2013).

[52] Y. Jiao, F. H. Stillinger, and S. Torquato, Phys. Rev. E 81, 041304 (2010).

[53] A. Donev, R. Connelly, F. H. Stillinger, and S. Torquato, Phys. Rev. E 75, 051304 (2007).

[54] A. Jaoshvili, A. Esakia, M. Porrati, and P. M. Chaikin, Phys. Rev. Lett. 104, 185501 (2010).

[55] E. Azema and F. Radjai, Phys. Rev. E 85, 031303 (2012).

[56] H. Ouadfel and L. Rothenburg, Mech. Mater. 33, 201 (2001).

[57] L. Rothenburg and R. J. Bathurst, Geotechnique 39, 601 (1989).

[58] C. Voivret, F. Radjaï, J.-Y. Delenne, and M. S. El Youssoufi, Phys. Rev. Lett. 102, 178001 (2009).

[59] N. Estrada, E. Azema, F. Radjai, and A. Taboada, Phys. Rev. E 84, 011306 (2011).

[60] F. Radjai, D. E. Wolf, M. Jean, and J. J. Moreau, Phys. Rev. Lett. 80, 61 (1998). 